\title{
Expansive automorphisms of totally disconnected, locally compact groups
}

\author{
Helge Glöckner and C.R.E. Raja
}

\begin{abstract}
We study automorphisms $\alpha$ of a totally disconnected, locally compact group $G$ which are expansive in the sense that $\bigcap_{n \in \mathbb{Z}} \alpha^{n}(U)=\{1\}$ for some identity neighbourhood $U \subseteq G$. Notably, we prove that the automorphism induced by $\alpha$ on a quotient group $G / N$ of $G$ modulo an $\alpha$ stable closed normal subgroup $N$ is always expansive. Further results involve the contraction groups $U_{\alpha}:=\left\{g \in G: \alpha^{n}(g) \rightarrow 1\right.$ as $\left.n \rightarrow \infty\right\}$. If $\alpha$ is expansive, then $U_{\alpha} U_{\alpha^{-1}}$ is an open identity neighbourhood in $G$. We give examples where $U_{\alpha} U_{\alpha^{-1}}$ fails to be a subgroup. However, $U_{\alpha} U_{\alpha^{-1}}$ is an $\alpha$-stable, nilpotent open subgroup of $G$ if $G$ is a closed subgroup of $\mathrm{GL}_{n}\left(\mathbb{Q}_{p}\right)$. Further results are devoted to the divisible and torsion parts of $U_{\alpha}$, and to the so-called "nub" $U_{0}=\overline{U_{\alpha}} \cap \overline{U_{\alpha^{-1}}}$ of an expansive automorphism.
\end{abstract}

Classification: 22D05 (primary); 22D45, 22E20, 37A25, 37P20 (secondary)

Key words: Totally disconnected group, locally compact group, locally profinite group, contraction group, expansive automorphism, contractive automorphism, tidy subgroup, Willis's theory, $p$-adic Lie group, finite depth, composition series, simple factor, BaumslagSolitar group, Schlichting completion, nub, closedness, normalizer

\section{Introduction and statement of results}

We consider automorphisms $\alpha: G \rightarrow G$ of a totally disconnected locally compact topological group $G$ which are expansive in the sense that $\bigcap_{n \in \mathbb{Z}} \alpha^{n}(U)=$ $\{1\}$ for some identity neighbourhood $U \subseteq G$. Expansive automorphisms of totally disconnected, compact groups were studied by [14], [22] and recently in [31]. The importance of expansive automorphisms for the theory of general automorphisms is highlighted by the fact that every automorphism $\alpha$ of a totally disconnected compact group $G$ is a projective limit

$$
\alpha=\lim _{\longleftarrow} \alpha_{j}
$$

of expansive automorphisms $\alpha_{j}$ of certain Hausdorff quotient groups $G / N_{j}$ of $G$ such that $G=\lim _{\longleftarrow} G / N_{j}$ (see [31]). Our goal is to improve the un- 
derstanding in the case of non-compact groups. Special cases of expansive automorphisms are automorphisms $\alpha: G \rightarrow G$ which are contractive in the sense that $\alpha^{n}(g) \rightarrow 1$ as $n \rightarrow \infty$ for each $g \in G$ (see Remark 1.10). The structure of totally disconnected, locally compact groups admitting contractive automorphisms was elucidated in [10] (building on earlier work like [24] and [26]), and the results obtained there can also be used as tools in the investigation of expansive automorphisms (as we shall see). Other key ingredients are the structure theory of totally disconnected, locally compact groups ([29], [30]), in which contractive automorphisms play an important role (as worked out in [1]).

Note that every automorphism $\alpha$ of a discrete group $G$ (e.g., $\alpha=\mathrm{id}_{G}$ ) is expansive (as we may choose $U=\{1\}$ then). Therefore discrete groups and their automorphisms are part of the theory of expansive automorphisms. As a consequence, groups permitting expansive automorphisms need not have any particular algebraic properties.

Our first main result generalizes [31, Proposition 6.1] (devoted to the case of compact groups). As usual, a subset $H \subseteq G$ is called $\alpha$-stable if $\alpha(H)=H$.

Theorem A. Let $\alpha: G \rightarrow G$ be an automorphism of a totally disconnected, locally compact group, $N \subseteq G$ be an $\alpha$-stable closed normal subgroup and $\bar{\alpha}: G / N \rightarrow G / N, g N \mapsto \alpha(g) N$ be the automorphism of $G / N$ induced by $\alpha$. Then $\alpha$ is expansive if and only if both $\left.\alpha\right|_{N}$ and $\bar{\alpha}$ are expansive.

Composition series play a central role in the study of contractive automorphisms [10]. In the case of expansive automorphisms, composition series need not exist. However, there are series which get as close as possible to such.

Theorem B. If $\alpha: G \rightarrow G$ is an expansive automorphism of a totally disconnected, locally compact group $G$, then there exist $\alpha$-stable closed subgroups

$$
G=G_{0} \supseteq G_{1} \supseteq \cdots \supseteq G_{n}=\{1\}
$$

of $G$ such that $G_{j}$ is normal in $G_{j-1}$ for $j \in\{1, \ldots, n\}$ and every $\alpha_{j}$-stable closed normal subgroup of $G_{j-1} / G_{j}$ is discrete or open, where $\alpha_{j}$ denotes the induced automorphism $G_{j-1} / G_{j} \rightarrow G_{j-1} / G_{j}, g G_{j} \mapsto \alpha(g) G_{j}$. Moreover, one can achieve that each of the quotient groups $G_{j-1} / G_{j}$ is discrete, abelian or topologically perfect. 
In addition, one may assume that all abelian, non-discrete factors $G_{j-1} / G_{j}$ are simple contraction groups with respect to the automorphism $\alpha_{j}$ or its inverse, or isomorphic to an infinite power $C_{p}^{\mathbb{Z}}$ of a cyclic group of prime order, endowed with the right-shift (cf. Remark 6.1 and Proposition 6.2). Topological perfectness means that the commutator group is dense. For second countable groups, more detailed information on the perfect factors is available (see Remark 4.2). The proofs of Theorem A and Theorem B hinge on the fact that there is a bound on the number of non-discrete factors in series for $(G, \alpha)$ (Proposition 2.7).

According to [30], a compact open subgroup $V \subseteq G$ is called tidy for $\alpha$ if it has the following properties:

T1 $V=V_{+} V_{-}$, where $V_{+}:=\bigcap_{n=0}^{\infty} \alpha^{n}(V)$ and $V_{-}:=\bigcap_{n=0}^{\infty} \alpha^{-n}(V)$;

T2 The $\alpha$-stable subgroups $V_{++}:=\bigcup_{n \in \mathbb{N}_{0}} \alpha^{n}\left(V_{+}\right)$and $\bigcup_{n \in \mathbb{N}_{0}} \alpha^{-n}\left(V_{-}\right)=$: $V_{--}$are closed in $G$.

Note that $V_{+} \subseteq \alpha\left(V_{+}\right) \subseteq \alpha^{2}\left(V_{+}\right) \subseteq \cdots$ and $V_{-} \subseteq \alpha^{-1}\left(V_{-}\right) \subseteq \alpha^{-2}\left(V_{-}\right) \subseteq \cdots$ here. Following [31], the intersection $U_{0}$ of all subgroups $V$ which are tidy for $\alpha$ is called the $n u b$ of $\alpha$; it is a compact, $\alpha$-stable subgroup of $G$.

We mention that a totally disconnected, locally compact group admitting an expansive automorphism $\alpha$ is always metrizable (cf. [15]) and has a second countable, $\alpha$-stable open subgroup (Lemma 1.1(a)).

If $\alpha: G \rightarrow G$ is an automorphism of a totally disconnected, locally compact group $G$, then

$$
U_{\alpha}:=\left\{g \in G: \alpha^{n}(g) \rightarrow 1 \text { as } n \rightarrow \infty\right\}
$$

is a subgroup of $G$ called the associated contraction group. In general, $U_{\alpha}$ need not be closed. However, if $\alpha$ is expansive, then the topology on $U_{\alpha}$ "can be made locally compact," i.e., it can be refined to a totally disconnected, locally compact group topology $\tau^{*}$ with respect to which $\left.\alpha\right|_{U_{\alpha}}$ is contractive (see [25, Proposition 9] for this fact, or our Lemma 2.3). In this way, the structure theory of locally compact contraction groups (see [24], 26] and [10]) becomes available. In particular, the set

$$
T_{\alpha}:=\operatorname{tor}\left(U_{\alpha}\right)
$$


of all torsion elements in the group $U_{\alpha}$ and the set

$$
D_{\alpha}:=\operatorname{div}\left(U_{\alpha}\right)
$$

of divisible elements are $\alpha$-stable closed subgroups of $\left(U_{\alpha}, \tau^{*}\right)$, and $\left(U_{\alpha}, \tau^{*}\right)=$ $D_{\alpha} \times T_{\alpha}$ internally as a topological group, if we endow $D_{\alpha}$ and $T_{\alpha}$ with the topology induced by $\left(U_{\alpha}, \tau^{*}\right)$ (see [10, Theorem B]).

Recall that the closure $\overline{U_{\alpha}}$ of the contraction group $U_{\alpha}$ in $G$ plays a role in the structure theory of totally disconnected, locally compact groups; for example, the scale of $\alpha^{-1}$ can be calculated on $\overline{U_{\alpha}}$ (see [1, Proposition 3.21 (3)]). Our next theorem provides information on $\overline{U_{\alpha}}$, and on the divisible part $D_{\alpha}$ of $U_{\alpha}$.

Theorem C. Let $G$ be a totally disconnected, locally compact group and $\alpha: G \rightarrow G$ be an automorphism such that $U_{\alpha}$ can be made locally compact (for example, any expansive automorphism). Then $\overline{U_{\alpha}}=D_{\alpha} \times \overline{T_{\alpha}}$ (internally) as a topological group, and $\overline{T_{\alpha}}=T_{\alpha} U_{0}$. In particular, $D_{\alpha}=\operatorname{div}\left(U_{\alpha}\right)$ is an $\alpha$ stable closed subgroup of $G$, and both the closure $\overline{T_{\alpha}}$ of $T_{\alpha}$ in $G$ and the nub $U_{0}$ centralize $D_{\alpha}$.

We mention that the nub $U_{0}$ of an expansive automorphism need not have an open normalizer in $G$ (Remark [5.2), in which case not both of $U_{\alpha}$ and $U_{\alpha^{-1}}$ normalize $U_{0}$.

Classes of examples are also considered. An analytic automorphism $\alpha$ of a Lie group $G$ over a totally disconnected local field $\mathbb{K}$ is expansive if and only if the associated Lie algebra automorphism $L(\alpha)$ is expansive, which means that none of its eigenvalues in an algebraic closure has absolute value 1 . The Lie algebra $L(G)$ of $G$ is then nilpotent (Proposition 7.1). In the case of $p$-adic Lie groups for a prime number $p$, we obtain:

Theorem D. Let $G$ be a p-adic Lie group which is linear in the sense that there exists an injective continuous homomorphism $G \rightarrow \mathrm{GL}_{n}\left(\mathbb{Q}_{p}\right)$ for some $n \in \mathbb{N}$. Let $\alpha: G \rightarrow G$ be an expansive automorphism. Then $G$ has an open $\alpha$-stable subgroup which is nilpotent.

If $\alpha: G \rightarrow G$ is an expansive automorphism of a totally disconnected, locally compact group $G$, then $U_{\alpha} U_{\alpha^{-1}}$ is an open subset of $G$ (see Proposition 1.1). In many examples, $U_{\alpha} U_{\alpha^{-1}}$ happens to be a subgroup of $G$ (for instance, for all expansive automorphisms of closed subgroups $G \subseteq \mathrm{GL}_{n}\left(\mathbb{Q}_{p}\right)$, 
see Proposition 7.8). However, this is not always so which could be seen from Remark 7.7. We also consider the localized completion $G_{p, q}$ of a BaumslagSolitar group $\operatorname{BS}(p, q)=\left\langle a, t: t a^{p} t^{-1}=a^{q}\right\rangle$ with primes $p \neq q$, as recently studied in [4]. Then $\operatorname{BS}(p, q) \subseteq G_{p, q}$. We show:

Theorem E. Let $\alpha: G_{p, q} \rightarrow G_{p, q}$ be conjugation by $t$. Then $\alpha$ is expansive but $U_{\alpha} U_{\alpha^{-1}}$ is not a subgroup of $G_{p, q}$.

Acknowledgement. The second author was supported by the German Academic Exchange Service (DAAD) and the second author wishes to thank DAAD and Institut für Mathematik, Universität Paderborn, Paderborn, Germany for providing the facilities during his stay.

\section{Preliminaries and basic facts}

We write $\mathbb{N}=\{1,2, \ldots\}, \mathbb{N}_{0}:=\mathbb{N} \cup\{0\}$ and $\mathbb{Z}:=\mathbb{N}_{0} \cup-\mathbb{N}$. If $J$ is a finite set, we let $\# J$ be its cardinality. We write $X \subseteq Y$ for inclusion of sets, while $X \subset Y$ means that $X$ is a proper subset of $Y$. As usual, we write $N \triangleleft G$ if $N$ is a normal subgroup of $G$. All topological groups considered in this article are assumed Hausdorff, and locally compact topological groups are simply called locally compact groups. Totally disconnected, locally compact non-discete topological fields (like the field of $p$-adic numbers) will be called local fields (see [27] for further information). See [3] and [23] for basic information on Lie groups over local (and more general complete ultrametric) fields (which we always assume finite-dimensional). If we say that $\alpha$ is an automorphism of a topological group, then we assume that both $\alpha$ and $\alpha^{-1}$ are continuous; similarly, both $\alpha$ and $\alpha^{-1}$ are assumed analytic if $\alpha$ is an automorphism of an analytic Lie group over a local field. We write $\operatorname{Aut}(G)$ for the group of all automorphisms of a topological group $G$. If a subgroup $N \subseteq G$ is stable under all $\alpha \in \operatorname{Aut}(G)$, then $N$ is called topologically characteristic. A topological group $G$ is called topologically perfect if its commutator group $[G, G]$ is dense in $G$. If $F$ is a finite group and $X$ a set, we write $F^{X}:=\prod_{x \in X} F$ for the direct power endowed with the compact product topology. By contrast, $F^{(X)} \subseteq F^{X}$ is the subgroup of all $\left(g_{x}\right)_{x \in X} \in F^{X}$ such that $g_{x}=1$ for all but finitely many $x \in X$. We shall always endow $F^{(X)}$ with the discrete topology. Surjective, open, continuous homomorphisms between topological groups are called quotient morphisms. 
If $\alpha: G \rightarrow G$ is an automorphism of a locally compact group, choose a Haar measure $\lambda$ on $G$ and define the module of $\alpha$ via

$$
\Delta_{G}(\alpha):=\lambda(\alpha(K)) / \lambda(K)
$$

for any compact subset $K \subseteq G$ with non-empty interior. If $\alpha: G \rightarrow G$ is an automorphism of a totally disconnected, locally compact group, we define the nub $U_{0}$, the contraction group $U_{\alpha}$ and its subgroups $T_{\alpha}$ and $D_{\alpha}$ as explained in the introduction. If $V \subseteq G$ is a compact open subgroup, we shall use the subgroups $V_{+}, V_{++}, V_{-}$and $V_{--}$defined there, and abbreviate

$$
V_{0}:=V_{+} \cap V_{-}=\bigcap_{n \in \mathbb{Z}} \alpha^{n}(V)
$$

We shall also need the so-called Levi factor

$$
M_{\alpha}:=\left\{g \in G:\left\{\alpha^{n}(g): n \in \mathbb{Z}\right\} \text { is relatively compact }\right\}
$$

it is known that $M_{\alpha}$ is an $\alpha$-stable closed subgroup of $G$ [1, p. 224]. The following lemma compiles basic facts concerning expansive automorphisms.

Lemma 1.1 If $\alpha$ is an expansive automorphism of a totally disconnected, locally compact group $G$, then the following holds:

(a) $G$ is metrizable and has an $\alpha$-stable, $\sigma$-compact open subgroup;

(b) $V_{--}=U_{\alpha}$ and $V_{++}=U_{\alpha^{-1}}$ for each compact open subgroup $V \subseteq G$ such that $V_{0}=\{1\}$;

(c) $G$ has a compact open subgroup $V$ such that $V=V_{+} V_{-}$and $V_{0}=\{1\}$;

(d) $U_{\alpha} U_{\alpha^{-1}}$ is open in $G$;

(e) $\left.\alpha\right|_{H}$ is expansive, for each $\alpha$-stable subgroup $H \subseteq G$.

Proof. (a) Because $\alpha$ is expansive, there exists an identity neighbourhood $V$ such that $\bigcap_{n \in \mathbb{Z}} \alpha^{n}(V)=\{1\}$. After shrinking $V$, we may assume that $V$ is compact. Because $V$ is compact and $\left(\bigcap_{k=-n}^{n} \alpha^{k}(V)\right)_{k \in \mathbb{N}}$ a decreasing sequence of closed identity neighbourhoods in $V$ with intersection $\{1\}$, the members of the sequence form a basis of identity neighbourhoods. Hence $G$ is metrizable. The subgroup of $G$ generated by $\bigcup_{n \in \mathbb{Z}} \alpha^{n}(V)$ is $\alpha$-stable, open and $\sigma$-compact. 
(b) By [1, Proposition 3.16], we have $V_{--}=U_{\alpha} V_{0}$ and thus $V_{--}=U_{\alpha}$. Likewise, $V_{++}=U_{\alpha^{-1}}$.

(c) and (d): Using expansiveness and van Dantzig's Theorem [11, Theorem 7.7], we find a compact open subgroup $W \subseteq G$ such that $\bigcap_{n \in \mathbb{Z}} \alpha^{n}(W)=$ $\{1\}$. By [29, Lemma 1], there exists $m \in \mathbb{N}$ such that $V:=\bigcap_{k=1}^{m} \alpha^{k}(W)$ satisfies $V=V_{+} V_{-}$. Then $V_{0} \subseteq W_{0}=\{1\}$ and thus $V_{0}=\{1\}$, proving (c). The latter entails $U_{\alpha}=V_{--}$and $U_{\alpha^{-1}}=V_{++}$, by (b). In particular, $V_{-} \subseteq U_{\alpha}$ and $V_{+} \subseteq U_{\alpha^{-1}}$, entailing that $V=V_{+} V_{-} \subseteq U_{\alpha} U_{\alpha^{-1}}$. Thus $U_{\alpha} U_{\alpha^{-1}}$ is an identity neighbourhood. Given $g \in U_{\alpha}$ and $h \in U_{\alpha^{-1}}$, the map $G \rightarrow G, x \mapsto g x h$ is a homeomorphism which takes $U_{\alpha} U_{\alpha^{-1}}$ onto itself and 1 to $g h$. Hence $U_{\alpha} U_{\alpha^{-1}}$ has $g h$ in its interior and thus $U_{\alpha} U_{\alpha^{-1}}$ is open.

(e) If $V \subseteq G$ is an identity neighbourhood with $\bigcap_{n \in \mathbb{Z}} \alpha^{n}(V)=\{1\}$, then $V \cap H$ is an identity neighbourhood in $H$ and $\bigcap_{n \in \mathbb{Z}} \alpha^{n}(V \cap H)=\{1\}$.

The first statement of Lemma 1.1(a) also follows from [15, Lemma 2.4].

1.2 Let $\alpha$ be an automorphism of a totally disconnected, locally compact group $G$ and $U_{0}$ be the nub of $\alpha$. The following facts are useful:

(a) The closure of $U_{\alpha}$ in $G$ is $\overline{U_{\alpha}}=U_{\alpha} U_{0}$ (see [1, Corollary 3.30] if $G$ is metrizable; the general case follows with [13]).

(b) $U_{0}=\overline{U_{\alpha}} \cap \overline{U_{\alpha^{-1}}}$ (see [1, Corollary 3.27] if $G$ is metrizable; the general case follows with [13]).

(c) $U_{0} \cap U_{\alpha}$ and $U_{0} \cap U_{\alpha^{-1}}$ are dense in $U_{0}$ (see [31, Theorem 4.1 (v) and Proposition $5.4(\mathrm{i})]$ ).

(d) If $N \subseteq G$ is a closed $\alpha$-stable subgroup, $q: G \rightarrow G / N$ the canonical quotient morphism and $\bar{\alpha}$ the automorphism of $G / N$ induced by $\alpha$, then $q\left(U_{\alpha}\right)=U_{\bar{\alpha}}$ (see [1, Theorem 3.8] if $G$ is metrizable, [13] in the general case).

(e) $U_{\alpha}$ is closed if and only if $U_{\alpha^{-1}}$ is closed, if and only if $G$ has small subgroups tidy for $\alpha$, i.e., every identity neighbourhood of $G$ contains some tidy subgroup (see [1, Theorem 3.32] if $G$ is metrizable; the general case can be deduced using the techniques from [13]).

(f) $P_{\alpha}:=\left\{g \in G: \alpha^{\mathbb{N} 0}(g)\right.$ is relatively compact $\}$ normalizes $U_{\alpha}$ (see [1, Proposition 3.4]). Hence $M_{\alpha}=P_{\alpha} \cap P_{\alpha^{-1}}$ and $U_{0} \subseteq M_{\alpha}$ normalize $U_{\alpha}$. 
The following results help to show that certain automorphisms are expansive.

Proposition 1.3 Let $\alpha$ be an automorphism of a totally disconnected, locally compact group $G$. Then the following holds:

(a) $\alpha$ is expansive if and only if its restriction $\left.\alpha\right|_{M_{\alpha}}$ to the Levi factor is expansive.

(b) If $U_{\alpha}$ is closed, then $\alpha$ is expansive if and only if $U_{\alpha} U_{\alpha^{-1}}$ is open in $G$, if and only if $M_{\alpha}$ is discrete.

Proof. (a) In view of Lemma 1.1(e), we only need to show that if $\left.\alpha\right|_{M_{\alpha}}$ is expansive, then so is $\alpha$. Let $P \subseteq M_{\alpha}$ be a compact, open identity neighbourhood such that $\bigcap_{n \in \mathbb{Z}} \alpha^{n}(P)=\{1\}$. There is a compact identity neighbourhood $Q \subseteq G$ such that $Q \cap M_{\alpha}=P$. If $g \in I:=\bigcap_{n \in \mathbb{Z}} \alpha^{n}(Q)$, then $\alpha^{n}(g) \in Q$ for each $n \in \mathbb{Z}$, whence $\alpha^{\mathbb{Z}}(g)$ is relatively compact and thus $g \in M_{\alpha}$. Hence $I \subseteq M_{\alpha}$. Since $I$ is $\alpha$-stable and $I \subseteq Q \cap M_{\alpha}=P$, we deduce that $I=\bigcap_{n \in \mathbb{Z}} \alpha^{n}(I) \subseteq \bigcap_{n \in \mathbb{Z}} \alpha^{n}(P)=\{1\}$. Thus $\bigcap_{n \in \mathbb{Z}} \alpha^{n}(Q)=\{1\}$ and thus $\alpha$ is expansive.

(b) If $\alpha$ is expansive, then $U_{\alpha} U_{\alpha^{-1}}$ is open by Lemma 1.1](d). If $U_{\alpha}$ is closed, then the set $U_{\alpha} M_{\alpha} U_{\alpha^{-1}}$ is open in $G$ and the product map

$$
U_{\alpha} \times M_{\alpha} \times U_{\alpha^{-1}} \rightarrow U_{\alpha} M_{\alpha} U_{\alpha^{-1}}, \quad(x, y, z) \mapsto x y z
$$

is a homeomorphism (by 1.2(e) above and part (f) from the theorem in [5]). Hence $U_{\alpha} U_{\alpha^{-1}} \cap M_{\alpha}=\{1\}$. If $U_{\alpha} U_{\alpha^{-1}}$ is open, this implies that $M_{\alpha}$ is discrete. If $M_{\alpha}$ is discrete, then $\left.\alpha\right|_{M_{\alpha}}$ is expansive and hence also $\alpha$, by (a).

1.4 If $\alpha$ is an automorphism of a totally disconnected, compact group $G$, then $U_{\alpha}$ and $U_{\alpha^{-1}}$ are normal in $G$ (since $G=M_{\alpha}=M_{\alpha^{-1}}$ in 1.2(f)), and hence so is the nub $U_{0}=\overline{U_{\alpha}} \cap \overline{U_{\alpha^{-1}}}$.

1.5 Recall that a group $G$ is called a torsion group of finite exponent if there exists $m \in \mathbb{N}$ such that $g^{m}=1$ for all $g \in G$. If such a group is a subgroup of a topological group $H$, then also $g^{m}=1$ for all $g$ in the closure $\bar{H}$ of $G$ in $H$, and thus also $\bar{H}$ is a torsion group of finite exponent.

1.6 Let $\alpha$ be an expansive automorphism of a totally disconnected, compact group $G$. Then the following holds: 
(a) The nub $U_{0}$ is open in $G$ (see [31, Lemma 5.1]), whence also $\overline{U_{\alpha}}$ and $\overline{U_{\alpha^{-1}}}$ are open in $G$ (by $\left.1.2(\mathrm{~b})\right)$.

(b) $G$ is a torsion group of finite exponent [By (a) and 1.4, $U_{0}$ is a normal subgroup and $G / U_{0}$ is finite, hence a torsion group of finite exponent. It therefore suffices to show that $U_{0}$ is a torsion group of finite exponent. This is immediate from [31, Proposition 4.4, Theorem 6.2 and Proposition 6.3]).

(c) Since $U_{\alpha}$ and $U_{\alpha^{-1}}$ are normal in $G$, the set $U_{\alpha} U_{\alpha^{-1}}$ is an open $\alpha$-stable subgroup of $G$ contained in $U_{0}$. Thus $U_{0}=U_{\alpha} U_{\alpha^{-1}}$ by [31, Corollary 4.3].

Lemma 1.7 Let $\alpha$ be an automorphism of a totally disconnected, locally compact group $G$ and $U_{0}$ be the nub of $\alpha$. Let $H \subseteq G$ be a closed, $\alpha$-stable subgroup. Then the following holds:

(a) Then nub of $\left.\alpha\right|_{H}$ is contained in $U_{0}$.

(b) If $U_{0} \subseteq H$, then the nub of $\left.\alpha\right|_{H}$ coincides with $U_{0}$.

Proof. (a) Let $W$ be the nub of $\left.\alpha\right|_{H}$. Using 1.2(b) twice, we deduce that

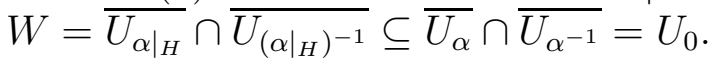

(b) Since $U_{0} \subseteq H$, we have $U_{\alpha} \cap U_{0} \subseteq U_{\alpha} \cap H=U_{\left.\alpha\right|_{H}}$ and thus $U_{0}=$ $\overline{U_{0} \cap U_{\alpha}} \subseteq \overline{U_{\left.\alpha\right|_{H}}}$ (using [1.2(c)). Likewise, $\bar{U}_{0} \subseteq \overline{U_{\left(\left.\alpha\right|_{H}\right)^{-1}}}$. Hence $U_{0} \subseteq \overline{U_{\left.\alpha\right|_{H}}} \cap$ $\overline{U_{\left(\left.\alpha\right|_{H}\right)^{-1}}}=W$. Since $W \subseteq U_{0}$ by (a), equality follows.

We shall also need certain facts concerning contractive automorphisms.

1.8 Let $\alpha$ be a contractive automorphism of a topological group $G$.

(a) If $G \neq\{1\}$, then $G$ is infinite and non-discrete. [If $x \in G \backslash\{1\}$, then $\alpha^{n}(x) \neq 1$ for all $n$ and $\alpha^{n}(x) \rightarrow 1$, entailing that the topological group $G$ is not discrete and hence infinite.]

(b) If $G$ is locally compact, then $\alpha$ is compactly contractive, i.e., for each identity neighbourhood $U \subseteq G$ and compact set $K \subseteq G$ there exists $m \in \mathbb{N}$ such that $\alpha^{n}(K) \subseteq U$ for all $n \geq m$ (see [24, Lemma 1.4 (iv)]). Moreover, $G$ is non-compact (unless $G=\{1\}$ ); see [24, 3.1].

1.9 Let $\alpha$ be a contractive automorphism of a totally disconnected, locally compact group $G$. Then the following holds: 
(a) If $G \neq\{1\}$, then $\Delta_{G}\left(\alpha^{-1}\right)$ is an integer $\geq 2$ (see [10, Proposition $\left.1.1(\mathrm{e})\right]$ ).

(b) If $G=G_{0} \supset G_{1} \supset G_{2} \supset \cdots \supset G_{n}=\{1\}$ is a series of $\alpha$-stable closed subgroups of $G$ such that $G_{j}$ is a proper normal subgroup of $G_{j-1}$ for all $j \in\{1, \ldots, n\}$, then $n$ is bounded by the number of prime factors of $\Delta_{G}\left(\alpha^{-1}\right)$ (see [10, Lemma 3.5]).

(c) $G=\operatorname{div}(G) \times \operatorname{tor}(G)$ as a topological group for a divisible torsion free group $\operatorname{div}(G)$ and a torsion group $\operatorname{tor}(G)$ of finite exponent (cf. [10, Theorem B]).

(d) If $N \subseteq G$ is an $\alpha$-stable closed normal subgroup, $q: G \rightarrow G / N$ the canonical quotient morphism and $\bar{\alpha}$ the automorphism of $G / N$ induced by $\alpha$, then $q(\operatorname{div}(G))=\operatorname{div}(G / N)$ and $q(\operatorname{tor}(G))=\operatorname{tor}(G / N)$. [The inclusions $q(\operatorname{div}(G)) \subseteq \operatorname{div}(G / N) q(\operatorname{tor}(G)) \subseteq \operatorname{tor}(G / N)$ are clear. Since $G / N=q(\operatorname{div}(G) \operatorname{tor}(G))=q(\operatorname{div}(G)) q(\operatorname{tor}(G))$ and $G / N=$ $\operatorname{div}(G / N) \times \operatorname{tor}(G / N)$, equality follows.]

Remark 1.10 If an automorphism $\alpha: G \rightarrow G$ of a totally disconnected, locally compact group is contractive, then it is also expansive. To see this, let $V \subseteq G$ be any compact neighbourhood of the identity. If $g \in G \backslash\{1\}$, then $G \backslash\{g\}$ is an identity neighbourhood, whence there exists $n \in \mathbb{N}$ such that $\alpha^{n}(V) \subseteq G \backslash\{g\}$ (see 1.8(b)). Thus $g \notin \alpha^{n}(V)$ and we have shown that $\bigcap_{n \in \mathbb{Z}} \alpha^{n}(V)=\{1\}$.

\section{Making contraction groups locally compact}

The problem of refining group topologies on contraction groups was studied by Siebert [25]. The following special case is useful for our purposes.

Definition 2.1 Let $G$ be a topological group, with topology $\tau$, and

$$
\alpha:(G, \tau) \rightarrow(G, \tau)
$$

be a contractive automorphism. We say that $(G, \alpha)$ (or simply $G$ ) can be made locally compact if there exists a locally compact topology $\tau^{*}$ on $G$ making it a topological group such that $\tau \subseteq \tau^{*}$ and $\alpha:\left(G, \tau^{*}\right) \rightarrow\left(G, \tau^{*}\right)$ is a contractive automorphism. The topology $\tau^{*}$ is unique (if it exists), as will be recalled presently. We write $G^{*}$ for $G$, endowed with the topology $\tau^{*}$. If $\tau$ is totally disconnected, then also $\tau^{*}$ is totally disconnected (as the inclusion map $G^{*} \rightarrow G$ is continuous). 
The following fact is also obtained in [25, Corollary 8] as part of a more general theory.

Lemma 2.2 $\tau^{*}$ is uniquely determined by the properties from Definition 2.1 .

Proof. Assume that $\hat{\tau}$ is a group topology on $G$ with the same properties as $\tau^{*}$. We show that the identity map

$$
\phi:(G, \hat{\tau}) \rightarrow\left(G, \tau^{*}\right), \quad x \mapsto x
$$

is continuous. Reversing the roles of $\hat{\tau}$ and $\tau^{*}$, also $\phi^{-1}$ will be continuous and thus $\hat{\tau}=\tau^{*}$. Because $\phi$ is a homomorphism, we need only prove its continuity at 1 . By local compactness, there exists a compact identity neighbourhood $V \subseteq(G, \hat{\tau})$. After replacing $V$ with the closure of its interior $V^{0}$, we may assume that $V^{0}$ is dense in $V$. Then $V$ is also compact in $(G, \tau)$. Let $U \subseteq\left(G, \tau^{*}\right)$ be an arbitrary identity neighbourhood, and $W \subseteq\left(G, \tau^{*}\right)$ be a compact identity neighbourhood such that $W W^{-1} \subseteq U$. Then $W$ is also compact in $(G, \tau)$, hence closed in $(G, \tau)$ and hence closed in $(G, \hat{\tau})$. Since $\alpha:\left(G, \tau^{*}\right) \rightarrow\left(G, \tau^{*}\right)$ is contractive, we have $G=\bigcup_{n \in \mathbb{N}_{0}} \alpha^{-n}(W)$. Hence $V$ is the countable union of the closed subsets $V \cap \alpha^{-n}(W)$, for $n \in \mathbb{N}_{0}$. By the Baire Category Theorem, there exists $m \in \mathbb{N}_{0}$ such that $V \cap \alpha^{-m}(W)$ has non-empty interior in $V$. Because $V^{0}$ is dense in $V$, we deduce that $\alpha^{-n}(W) \cap$ $V^{0}$ has non-empty interior in $V^{0}$, and so $W$ has non-empty interior $W^{0}$ in $(G, \hat{\tau})$. Then $W^{0}\left(W^{0}\right)^{-1}$ is an identity neighbourhood in $(G, \hat{\tau})$ and hence $U$ is an identity neighbourhood in $(G, \hat{\tau})$. Since $U=\phi^{-1}(U)$, we see that $\phi$ is continuous at 1 .

See also [25, Proposition 9] for the following fact.

Lemma 2.3 Let $G$ be a totally disconnected, locally compact group. If an automorphism $\alpha: G \rightarrow G$ is expansive, then $\left(U_{\alpha},\left.\alpha\right|_{U_{\alpha}}\right)$ and $\left(U_{\alpha^{-1}},\left.\alpha^{-1}\right|_{U_{\alpha^{-1}}}\right)$ can be made locally compact.

Proof. Let $V \subseteq G$ be a compact open subgroup such that $\bigcap_{n \in \mathbb{Z}} \alpha^{n}(V)=$ $\{1\}$. Then $\alpha^{n}\left(V_{-}\right)=V_{-} \cap \bigcap_{k=1}^{n} \alpha^{k}(V)$ is open in $V_{-}$for each $n \in \mathbb{N}_{0}$. Since $V_{-}$is compact and $\bigcap_{n \in \mathbb{N}_{0}} \alpha^{n}\left(V_{-}\right)=\bigcap_{n \in \mathbb{Z}} \alpha^{n}(V)=\{1\}$, the open subgroups $\alpha^{n}\left(V_{-}\right)$form a basis of identity neighbourhoods in $V_{-}$, for $n \in \mathbb{N}_{0}$. If $n \in \mathbb{N}$ and $g \in U_{\alpha}=V_{--}=\bigcup_{k \in \mathbb{N}_{0}} \alpha^{-k}\left(V_{-}\right)$(see Proposition 1.1(c)), then $g \in \alpha^{-k}\left(V_{-}\right)$for some $k \in \mathbb{N}_{0}$ and $\alpha^{n}\left(V_{-}\right)$is an open subgroup of the topological group $\alpha^{-k}\left(V_{-}\right)$. Hence, there exists $m \in \mathbb{N}_{0}$ such that 
$g \alpha^{m}\left(V_{-}\right) g^{-1} \subseteq \alpha^{n}\left(V_{-}\right)$. By the preceding, there exists a group topology $\tau^{*}$ on $U_{\alpha}$ for which $\left\{\alpha^{n}\left(V_{-}\right): n \in \mathbb{N}_{0}\right\}$ is a basis of identity neighbourhoods. Thus $V_{-}$is an open subgroup of $\left(U_{\alpha}, \tau^{*}\right)$, and the latter group induces the given compact topology on $V_{-}$(as $\left\{\alpha^{n}\left(V_{-}\right): n \in \mathbb{N}_{0}\right\}$ is a basis of identity neighbourhoods for both topologies on $\left.V_{-}\right)$. Thus $\left(U_{\alpha}, \tau^{*}\right)$ is a totally disconnected, locally compact group and $\alpha$ is still continuous (being continuous on the open subgroup $V_{-}$) as well as $\alpha^{-1}$ (being continuous on the subgroup $\alpha\left(V_{-}\right)$which is open in $\left.V_{-}\right)$. Since $U_{\alpha}=\bigcup_{n \in \mathbb{N}_{0}} \alpha^{-n}\left(V_{-}\right)$and $\left(\alpha^{n}\left(V_{-}\right)\right)_{n \in \mathbb{N}_{0}}$ is a basis of identity neighbourhoods, it readily follows that the automorphism $\alpha$ of $\left(U_{\alpha}, \tau^{*}\right)$ is contractive.

Remark 2.4 $U_{\alpha}$ can also be made locally compact if $\alpha$ is an arbitrary (not necessarily expansive) analytic automorphism of a Lie group $G$ over a local field (see Proposition 13.3 (b) in the extended preprint version of [7]).

Example 2.5 The right-shift $\alpha$ is an automorphism of the compact group $G:=\left(\mathbb{Z}_{p}\right)^{\mathbb{Z}}$, where $\mathbb{Z}_{p}$ is the additive group of $p$-adic integers. The contraction group $U_{\alpha}$ is non-trivial, as it is the group of all $\left(z_{n}\right)_{n \in \mathbb{Z}}$ such that $z_{n} \rightarrow 0$ as $n \rightarrow-\infty$. Then $U_{\alpha}$ cannot be locally compact, because $\operatorname{tor}\left(U_{\alpha}\right) \subseteq \operatorname{tor}(G)=$ $\{0\}$ and $\operatorname{div}\left(U_{\alpha}\right) \subseteq \operatorname{div}(G)=\{0\}$. Thus, if $U_{\alpha}$ could be made locally compact, then we would have $U_{\alpha}=\operatorname{div}\left(U_{\alpha}\right)+\operatorname{tor}\left(U_{\alpha}\right)=\{0\}$, contradicting $U_{\alpha} \neq\{0\}$.

Lemma 2.6 Let $G$ be a topological group and $\alpha: G \rightarrow G$ a contractive automorphism such that $(G, \alpha)$ can be made locally compact. Then we have:

(a) $\left(H,\left.\alpha\right|_{H}\right)$ can be made locally compact for each closed $\alpha$-stable subgroup $H$ of $G\left(\right.$ or $\left.G^{*}\right)$, and $H^{*}$ carries the topology induced by $G^{*}$.

(b) If $\phi: G \rightarrow H$ is a continuous homomorphism to a topological group $H$ admitting an automorphism $\beta: H \rightarrow H$ such that $\beta \circ \phi=\phi \circ \alpha$, then $\left.\beta\right|_{\phi(G)}$ is contractive, $\phi(G)$ can be made locally compact and

$$
G^{*} \rightarrow \phi(G)^{*}, \quad x \mapsto \phi(x)
$$

is a topological quotient map.

Proof. (a) Let $\tau$ and $\tau^{*}$ be as in Definition 2.1. Since $\tau \subseteq \tau^{*}, H$ is closed in $G^{*}$ (in either case) and hence $H$ is a locally compact group in the topology $\sigma$ on $H$ induced by $G^{*}$, which is finer than the topology induced by $G$ and turns $\left.\alpha\right|_{H}$ into a contractive automorphism of $(H, \sigma)$. Thus $H^{*}=(H, \sigma)$. 
(b) Let $\sigma$ be the topology on $\phi(G)$ turning $G^{*} \rightarrow(\phi(G), \sigma), x \mapsto \phi(x)$ into a quotient map. Then $\sigma$ is finer than the topology induced on $\phi(G)$ by $H$. Moreover, $(\phi(G), \sigma) \cong G^{*} / \operatorname{ker} \phi$ is locally compact. Since $\left.\beta\right|_{\phi(G)} \circ \phi=$ $\phi \circ \alpha: G^{*} \rightarrow(\phi(G), \sigma)$ is continuous, the map $\left.\beta\right|_{\phi(G)}$ is continuous with respect to the quotient topology $\sigma$. Also $\left(\left.\beta\right|_{\phi(G)}\right)^{-1}$ is continuous, by an analogous argument. Finally, $\left.\beta\right|_{\phi(G)}:(\phi(G), \sigma) \rightarrow(\phi(G), \sigma)$ is contractive: Since $\beta^{n} \circ \phi=\phi \circ \alpha^{n}$, this follows from the facts that $\alpha: G^{*} \rightarrow G^{*}$ is contractive and $\phi: G^{*} \rightarrow \phi(G)^{*}$ is continuous.

The following observation is crucial for many of our arguments.

Proposition 2.7 Let $\alpha$ be an expansive automorphism of a totally disconnected, locally compact group $G$ and

$$
G=G_{0} \supseteq G_{1} \supseteq \cdots \supseteq G_{n}
$$

be $\alpha$-stable closed subgroups of $G$ such that $G_{j}$ is normal in $G_{j-1}$ for all $j \in\{1, \ldots, n\}$. Let $J$ be the set of all $j \in\{1, \ldots, n\}$ such that $G_{j-1} / G_{j}$ is not discrete. Then

$$
\# J \leq \ell_{\alpha}+\ell_{\alpha^{-1}}
$$

where $\ell_{\alpha}$ is the number of prime factors of $\Delta_{U_{\alpha}^{*}}\left(\left.\alpha^{-1}\right|_{U_{\alpha}^{*}}\right)$ and $\ell_{\alpha^{-1}}$ is the number of prime factors of $\Delta_{U_{\alpha}^{*}-1}\left(\left.\alpha\right|_{U^{*}-1} ^{*}\right)$.

Proof. Let $J_{\alpha}$ (resp., $J_{\alpha^{-1}}$ ) be the set of all $j \in\{1, \ldots, n\}$ such that $U_{\alpha} \cap G_{j} \subset$ $U_{\alpha} \cap G_{j-1}$ (resp., $U_{\alpha^{-1}} \cap G_{j} \subset U_{\alpha^{-1}} \cap G_{j-1}$ ). If $j \in\{1, \ldots, n\} \backslash\left(J_{\alpha} \cup J_{\alpha^{-1}}\right)$, then $U_{\alpha} \cap G_{j}=U_{\alpha} \cap G_{j-1}$ and $U_{\alpha^{-1}} \cap G_{j}=U_{\alpha^{-1}} \cap G_{j-1}$. Since $\alpha$ is expansive, $\left(U_{\alpha} \cap G_{j-1}\right)\left(U_{\alpha^{-1}} \cap G_{j-1}\right)$ is open in $G_{j-1}$ (see 1.1 (d) and (e)). We deduce that $G_{j}$ is open in $G_{j-1}$ and thus $j \notin J$. Hence $J \subseteq J_{\alpha} \cup J_{\alpha^{-1}}$, whence $\# J \leq \# J_{\alpha}+\# J_{\alpha^{-1}} \leq \ell_{\alpha}+\ell_{\alpha^{-1}}$ (using [1.9(b) in the last step).

We shall use a simple fact.

Lemma 2.8 . Let $K$ be a compact group and $D \subseteq K$ be a subgroup which is divisible. Then also the closure $\bar{D}$ is divisible. If $K$ is totally disconnected, then $D=\{1\}$.

Proof. For each $m \in \mathbb{N}$, the map $f_{m}: \bar{D} \rightarrow \bar{D}, g \mapsto g^{m}$ is continuous and hence has compact image. As the image contains $D$ by hypothesis, we see that $f_{m}(\bar{D})=\bar{D}$. Thus $\bar{D}$ is divisible. 
If $K$ is totally disconnected, then $K$ is a pro-finite group. In particular, the homomorphisms $f: K \rightarrow F$ to finite groups $F$ separate points on $\bar{D}$. But each $f(\bar{D})$ is both finite and divisible and therefore the trivial group. Hence also $\bar{D}=\{1\}$.

Lemma 2.9 Let $\alpha$ be an automorphism of a totally disconnected, locally compact group $G$. If $U_{\alpha}$ can be made locally compact (e.g., if $\alpha$ is expansive), then the following holds:

(a) $U_{\alpha} \cap U_{0}=T_{\alpha} \cap U_{0}$;

(b) $U_{0}=\overline{T_{\alpha} \cap U_{0}}$;

(c) $\overline{T_{\alpha}} \cap U_{\alpha}=T_{\alpha}$;

(d) $\overline{T_{\alpha}}=T_{\alpha} U_{0}$.

If both $U_{\alpha}$ and $U_{\alpha^{-1}}$ can be made locally compact, then we also have:

(e) $U_{0}=\overline{T_{\alpha}} \cap \overline{T_{\alpha^{-1}}}$.

Proof. (a) By Lemma 2.6(a), $U_{\alpha} \cap U_{0}$ can be made locally compact. Thus $\alpha$ restricts to a contractive automorphism $\beta$ of $\left(U_{\alpha} \cap U_{0}\right)^{*}$, enabling us to write $\left(U_{\alpha} \cap U_{0}\right)^{*}=D_{\beta} T_{\beta}$. Since $U_{0}$ is compact and totally disconnected, its divisible subgroup $D_{\beta}$ has to be trivial, by Lemma 2.8. Thus $U_{\alpha} \cap U_{0}=U_{\beta}=T_{\beta} \subseteq T_{\alpha}$ and hence $U_{\alpha} \cap U_{0}=T_{\alpha} \cap U_{0}$.

(b) Since $U_{0}=\overline{U_{\alpha} \cap U_{0}}$ (see $1.2(\mathrm{c})$ ), the assertion is immediate from (a).

(c) $T_{\alpha} \subseteq \overline{T_{\alpha}} \cap U_{\alpha}$ is trivial. Because $T_{\alpha}$ is a torsion group of finite exponent (see $1.9(\mathrm{c}))$, also $\overline{T_{\alpha}}$ is a torsion group, see 1.5. Let $\beta$ the restriction of $\alpha$ to the closed $\alpha$-stable subgroup $\overline{T_{\alpha}} \cap U_{\alpha}^{*}$ of $U_{\alpha}^{*}$. Then $\overline{T_{\alpha}} \cap U_{\alpha}=D_{\beta} T_{\beta}$. Since $D_{\beta}$ is torsion-free (see $1.9(\mathrm{c})$ ) and $\overline{T_{\alpha}}$ a torsion group, $D_{\beta}=\{1\}$ follows. Thus $\overline{T_{\alpha}} \cap U_{\alpha}=T_{\beta} \subseteq T_{\alpha}$.

(d) We have $\overline{T_{\alpha}} \subseteq \overline{U_{\alpha}}=U_{\alpha} U_{0}$ (see 1.2 (a)). Since $U_{0} \subseteq \overline{T_{\alpha}}$ by (b), we deduce that $\overline{T_{\alpha}}=\left(U_{\alpha} \cap \overline{T_{\alpha}}\right) U_{0}=T_{\alpha} U_{0}$ (using (c) for the last equality).


$\overline{T_{\alpha^{-1}} \cap U_{0}} \subseteq \overline{T_{\alpha}} \cap \overline{T_{\alpha^{-1}}}$ (using (b)).

Lemma 2.10 Let $\alpha$ be an automorphism of a locally compact group $G$ and $H \subseteq G$ be an $\alpha$-stable subgroup such that $\left.\alpha\right|_{H}$ is contractive. Then the closure $\bar{H} \subseteq G$ is $\sigma$-compact. 
Proof. Let $K \subseteq \bar{H}$ be a compact identity neighbourhood. Then $\bigcup_{n \in \mathbb{Z}} \alpha^{n}(K)$ is a $\sigma$-compact subset of $\bar{H}$ and generates a $\sigma$-compact subgroup $S$ of $\bar{H}$. Since $S$ is an $\alpha$-stable open subgroup of $\bar{H}$ and $\left.\alpha\right|_{H}$ is contractive, we have $H \subseteq S$ and thus $S=\bar{H}$ (since $S$ is closed). Hence $\bar{H}=S$ is $\sigma$-compact.

\section{Proof of Theorem A}

Let $G$ be a totally disconnected, locally compact group, $\alpha$ be an expansive automorphism of $G$ and $N \subseteq G$ be an $\alpha$-stable, closed normal subgroup. Let $q: G \rightarrow G / N$ be the canonical quotient morphism and $\bar{\alpha}$ be the automorphism of $G / N$ induced by $\alpha$ (determined by $\bar{\alpha} \circ q=q \circ \alpha$ ). Then $\left.\alpha\right|_{N}$ is expansive, by Lemma 1.1(e). We show that also $\bar{\alpha}$ is expansive. By Proposition 1.3 (a), we need only show that $\bar{\alpha}$ restricts to an expansive automorphism of $M_{\bar{\alpha}}$. After replacing $G$ with $q^{-1}\left(M_{\bar{\alpha}}\right)$ (which is closed since $M_{\bar{\alpha}}$ is closed), we may assume that $G / N=M_{\bar{\alpha}}$. Let $U$ be a subgroup of $G / N$ tidy for $\bar{\alpha}$. Then $U=U_{+}=U_{-}$as $\bar{\alpha}^{\mathbb{Z}}(g)$ is relatively compact for each $g \in U$ (cf. 29, Lemma 9]) and thus $U$ is an $\bar{\alpha}$-stable, compact open subgroup of $G / N$. After replacing $G$ with $q^{-1}(U)$, we may assume that $G / N$ is compact. Using [31, Proposition 5.1] and the metrizability of $G / N$, we find a descending sequence $\left(H_{n}\right)_{n \in \mathbb{N}}$ of $\bar{\alpha}$-stable closed normal subgroups $H_{n}$ of $G / N$ such that $\bar{\alpha}$ induces an expansive automorphism $\alpha_{n}$ on $(G / N) / H_{n}$ for each $n \in \mathbb{N}$ and $G / N$ is the projective limit $G / N=\lim (G / N) / H_{n}$. Set $L_{n}:=q^{-1}\left(H_{n}\right)$; then $\left(L_{n}\right)_{n \in \mathbb{N}}$ is a descending sequence of $\alpha$-stable closed normal subgroups of $G$, with $\bigcap_{n \in \mathbb{N}} L_{n}=N$.

There exists $m \in \mathbb{N}$ such that $L_{n}$ is open in $L_{m}$ for all $n \geq m$. Indeed, if this was wrong, we could find a subsequence $\left(L_{n_{k}}\right)_{k \in \mathbb{N}}$ such that, for each $k \in \mathbb{N}$, the normal subgroup $L_{n_{k+1}}$ is not open in $L_{n_{k}}$. This contradicts Proposition 2.7.

After passing to a subsequence, we may assume that $L_{n}$ is open in $L_{1}$ for each $n \in \mathbb{N}$. Hence $L_{n}$ contains both $U_{\alpha} \cap L_{1}$ and $U_{\alpha^{-1}} \cap L_{1}$. As a consequence, $N=\bigcap_{n \in \mathbb{N}} L_{n}$ contains both $U_{\alpha} \cap L_{1}$ and $U_{\alpha^{-1}} \cap L_{1}$. Hence $N$ is open in $L_{1}$ (and in each $\left.L_{n}\right)$, using that $\left.\alpha\right|_{L_{1}}$ is expansive and thus $\left(U_{\alpha} \cap L_{1}\right)\left(U_{\alpha^{-1}} \cap L_{1}\right)$ an open subset of $L_{1}$ (see 1.1 (d) and (e)). This implies that the compact group $H_{1} \cong L_{1} / N$ is discrete and hence a finite group. Since $H_{1} \supseteq H_{2} \supseteq \cdots$ with $\bigcap_{n \in \mathbb{N}} H_{n}=\{1\}$, we deduce that $H_{n}=\{1\}$ for some $n$. Since $\bar{\alpha}$ cor- 
responds to the expansive automorphism $\alpha_{n}$ on $(G / N) / H_{n} \cong G / N$, we see that $\bar{\alpha}$ is expansive.

Conversely, assume that both $\left.\alpha\right|_{N}$ and $\bar{\alpha}: G / N \rightarrow G / N$ are expansive 11 Then there is an open identity neighbourhood $P \subseteq G / N$ such that $\bigcap_{n \in \mathbb{Z}} \bar{\alpha}^{n}(P)=$ $\{1\}$, and an open identity neighbourhood $Q \subseteq N$ such that $\bigcap_{n \in \mathbb{Z}} \alpha^{n}(Q)=$ $\{1\}$. After shrinking $Q$, we may assume that $Q \subseteq q^{-1}(P)$. Then $Q=N \cap V$ for some open identity neighbourhood $V \subseteq G$. After replacing $V$ with $V \cap$ $q^{-1}(P)$, we may assume that $V \subseteq q^{-1}(P)$. We have $N=q^{-1}\left(\bigcap_{n \in \mathbb{Z}} \bar{\alpha}^{n}(P)\right)=$ $\bigcap_{n \in \mathbb{Z}} \alpha^{n}\left(q^{-1}(P)\right)$, entailing that $I:=\bigcap_{n \in \mathbb{Z}} \alpha^{n}(V)$ is an $\alpha$-stable subset of $N$. Since $I=I \cap N \subseteq V \cap N=Q$, we deduce that $I=\bigcap_{n \in \mathbb{Z}} \alpha^{n}(I) \subseteq$ $\bigcap_{n \in \mathbb{Z}} \alpha^{n}(Q)=\{1\}$. Hence $I=\{1\}$ and $\alpha$ is expansive.

\section{Proof of Theorem B}

The following lemma is useful.

Lemma 4.1 Let $\alpha$ be an expansive automorphism of a totally disconnected, locally compact group $G$ such that every $\alpha$-stable, closed normal subgroup $N \subseteq G$ is open or discrete. Let $C \subseteq G$ be the topologically characteristic subgroup 2 of $G$ generated by $U_{\alpha} \cup U_{\alpha^{-1}}$. Let $\overline{[C, C]}$ be the closure of the commutator group of $C$. Then $C$ is an open normal subgroup of $G$, and one of the following cases occurs:

(a) $\overline{[C, C]}$ is open in $G$, in which case $C=\overline{[C, C]}$ is topologically perfect.

(b) $\overline{[C, C]}$ is discrete.

Proof. $C$ is an open subgroup of $G$ as it contains the open set $U_{\alpha} U_{\alpha^{-1}}$ (see $1.1(\mathrm{~d})$ ). The closed subgroup $\overline{[C, C]}$ is topologically characteristic in $C$, whence it is topologically characteristic in $G$ and hence $\alpha$-stable and normal. Therefore $\overline{[C, C]}$ is open or discrete. If $\overline{[C, C]}$ is open, then it contains $U_{\alpha} \cup U_{\alpha^{-1}}$. Hence $\overline{[C, C]}=C$, using that $C$ is the smallest topologically

\footnotetext{
${ }^{1}$ Compare also [31, Proposition 6.1 ]. The compactness of $G$ assumed there is inessential for this part of the proof of [31, Proposition 6.1].

${ }^{2}$ Thus $C$ is the subgroup generated by $\bigcup_{\beta \in \operatorname{Aut}(G)} \beta\left(U_{\alpha} \cup U_{\alpha^{-1}}\right)$.
} 
characteristic subgroup of $G$ which contains $U_{\alpha} \cup U_{\alpha^{-1}}$.

Proof of Theorem B. Define $\ell_{\alpha}$ and $\ell_{\alpha^{-1}}$ as in Proposition 2.7. For every series

$$
\Sigma: G=G_{0} \triangleright G_{1} \triangleright \cdots \triangleright G_{n}=\{1\}
$$

of $\alpha$-stable closed subgroups of $G$, let $J_{\Sigma}$ be the set of all $j \in\{1, \ldots, n\}$ such that $G_{j-1} / G_{j}$ is not discrete. Then $J_{\Sigma} \leq \ell_{\alpha}+\ell_{\alpha^{-1}}$, by Proposition 2.7 , entailing that the maximum

$$
m:=\max _{\Sigma} J_{\Sigma}
$$

over all series $\Sigma$ exists. Let $\Sigma: G=G_{0} \triangleright G_{1} \triangleright \cdots \triangleright G_{n}=\{1\}$ be a series with $J_{\Sigma}=m$. Let $N \subseteq G_{j-1}$ be an $\alpha$-stable closed normal subgroup with $G_{j} \subseteq N$. If neither $G_{j-1} / N$ nor $N / G_{j}$ was discrete, we would have $J_{\Sigma \cup\{N\}}=J_{\Sigma}+1$, a contradiction. Thus $N$ will be open in $G_{j-1}$ or $G_{j}$ open in $N$.

For $j \in J_{\Sigma}$, let $q_{j}: G_{j-1} \rightarrow G_{j-1} / G_{j}$ be the canonical quotient morphism, $\alpha_{j}: G_{j-1} / G_{j} \rightarrow G_{j-1} / G_{j}$ be the automorphism induced by $\alpha$ and $C_{j} \subseteq$ $G_{j-1} / G_{j}$ be the topologically characteristic subgroup generated by $U_{\alpha_{j}} \cup$ $U_{\alpha_{j}^{-1}}$. If $\overline{\left[C_{j}, C_{j}\right]}$ is open in $G_{j-1} / G_{j}$, define $M_{j}:=N_{j}:=q_{j}^{-1}\left(C_{j}\right)$; thus $G_{j-1} / M_{j} \cong\left(G_{j-1} / G_{j}\right) / C_{j}$ and $M_{j} / N_{j}=\{1\}$ are discrete and $N_{j} / G_{j} \cong C_{j}$ is topologically perfect. If $\overline{\left[C_{j}, C_{j}\right]}$ is discrete, we define $M_{j}:=q_{j}^{-1}\left(C_{j}\right)$ and $N_{j}:=q_{j}^{-1}\left(\overline{\left[C_{j}, C_{j}\right]}\right)$ (by Lemma 4.1, only these two cases can occur). Then $G_{j-1} / M_{j} \cong\left(G_{j-1} / G_{j}\right) / C_{j}$ is discrete, $M_{j} / N_{j} \cong C_{j} / \overline{\left[C_{j}, C_{j}\right]}$ is abelian and $N_{j} / G_{j} \cong \overline{\left[C_{j}, C_{j}\right]}$ is discrete. Hence

$$
\Sigma^{\prime}:=\Sigma \cup \bigcup_{j \in J_{\Sigma}}\left\{M_{j}, N_{j}\right\}
$$

is a series of $\alpha$-stable closed subnormal subgroups such that all non-discrete subfactors are abelian or topologically perfect. Since $\# J_{\Sigma^{\prime}}=\# J_{\Sigma}$ is maximal, all non-discrete subfactors of $\Sigma^{\prime}$ have the property that all stable closed normal subgroups are open or discrete.

Using the recent theory of elementary groups [28, slightly more detailed information on the factor groups can be obtained, in the case of second countable groups. Recall that the class of elementary groups is the smallest class of totally disconnected, second countable, locally compact groups that 
contains all countable discrete groups and all second countable pro-finite groups, and is closed under extensions as well as countable increasing unions. A totally disconnected, second countable, locally compact group $G$ is called elementary-free if all of its elementary closed normal subgroups and all of its elementary Hausdorff quotient groups are trivial [28, Definition 7.14]. If $\alpha$ is an expansive automorphism of a totally disconnected, locally compact non-trivial group $G$ and $G$ does not have closed $\alpha$-stable subgroups except for $G$ and $\{1\}$, then $(G, \alpha)$ is called a simple expansion group. Note that if $G$ is a non-trivial elementary-free group and $\alpha$ an expansive automorphism of $G$ such that every $\alpha$-stable closed normal subgroup of $G$ is discrete or open, then $(G, \alpha)$ is a simple expansion group. We remark:

Remark 4.2 If $G$ is second countable in Theorem B, then one can achieve there that each of the quotient groups $G_{j-1} / G_{j}$ is discrete, abelian, both topologically perfect and elementary, or an elementary-free simple expansion group.

In fact, let us consider a topologically perfect factor $Q:=G_{j-1} / G_{j}$ in a series all of whose factors are discrete, abelian, or topologically perfect, and which has a maximum number of non-discrete factors. By [28, Theorem 7.15], there are topologically characteristic, closed subgroups $D_{1}$ and $D_{2}$ of $Q$ such that $Q \supseteq D_{1} \supseteq D_{2} \supseteq\{1\}$ and, moreover, both $D_{2}$ and $Q / D_{1}$ are elementary and $D_{1} / D_{2}$ is elementary-free. Let $N_{1}$ and $N_{2}$ be the pre-images of $D_{1}$ and $D_{2}$, respectively, under the quotient morphism $G_{j-1} \rightarrow G_{j-1} / G_{j}$. Then $N_{1}$ and $N_{2}$ are $\alpha$-stable closed normal subgroups of $G_{j-1}$, and $G_{j-1} \triangleright N_{1} \triangleright N_{2} \triangleright G_{j}$.

Case 1: If $D_{1} / D_{2}$ is non-trivial, then the elementary-free group $N_{1} / N_{2} \cong$ $D_{1} / D_{2}$ is non-discrete (as it would be elementary otherwise), whence $N_{2}$ is not open in $N_{1}$. Hence $N_{2}$ is not open in $G_{j-1}$ and hence $N_{2} / G_{j}$ is discrete (by maximailty of the number of non-discrete factors). Again by maximality, $G_{j-1} / N_{1}$ is discrete and $N_{1} / N_{2}$ does not have closed normal subgroups stable under the induced expansive automorphism other than open or discrete subgroups. So, the elementary-free group $N_{1} / N_{2}$ is a simple expansion group.

Case 2: If $D_{1} / D_{2}$ is trivial, then the topologically perfect group $G_{j-1} / G_{j}=$ $Q \triangleright D_{1}=D_{2} \triangleright\{1\}$ is elementary, as any extension of elementary groups. 


\section{Proof of Theorem C}

Since $U_{0} \subseteq \overline{U_{\alpha}}$, we may replace $G$ with $\overline{U_{\alpha}}$ without changing the nub (see 1.7 ), or $\overline{T_{\alpha}}$, or $D_{\alpha}$. We may therefore assume that $U_{\alpha}$ is dense in $G$. Since $U_{0}$ normalizes $U_{\alpha}$ (see 1.2(f)), $U_{\alpha}$ is a normal subgroup of $G=U_{\alpha} U_{0}$ (exploiting $1.2(\mathrm{a}))$. Hence also the characteristic subgroups $D_{\alpha}$ and $T_{\alpha}$ of $U_{\alpha}$ are normal in $G$. Therefore also $\overline{T_{\alpha}}$ is normal in $G$. Since $\overline{T_{\alpha}}$ is a torsion group (see 1.9 (c) and 1.5) and $D_{\alpha}$ torsion-free (see $1.9(\mathrm{c})$ ), we see that $D_{\alpha} \cap \overline{T_{\alpha}}=\{1\}$. Moreover, using that $T_{\alpha} U_{0}=\overline{T_{\alpha}}$ by Lemma 2.9(d), we obtain $G=U_{\alpha} U_{0}=$ $D_{\alpha} T_{\alpha} U_{0}=D_{\alpha} \overline{T_{\alpha}}$. Hence $G=D_{\alpha} \times \overline{T_{\alpha}}$ as an abstract group. In particular, $D_{\alpha}$ centralizes $\overline{T_{\alpha}}$. Thus $D_{\alpha}$ also centralizes $U_{0} \subseteq \overline{T_{\alpha}}$. Since $\overline{T_{\alpha}}$ is $\sigma$-compact by Lemma 2.10, also $D_{\alpha}^{*} \times \overline{T_{\alpha}}$ is a $\sigma$-compact locally compact group (writing $D_{\alpha}^{*}$ for $D_{\alpha}$, endowed with the locally compact topology induced by $U_{\alpha}^{*}$ ). Because also $G$ is locally compact and the product map $\pi: D_{\alpha}^{*} \times \overline{T_{\alpha}} \rightarrow G$, $(x, y) \mapsto x y$ is a continuous isomorphism of abstract groups, we deduce with $[11,5.29]$ that $\pi$ is an isomorphism of topological groups. Hence $G=D_{\alpha} \times \overline{T_{\alpha}}$ (internally). In particular, $D_{\alpha}$ is closed in $G$.

Corollary 5.1 Let $G$ be a totally disconnected, locally compact group and $\alpha$ be an automorphism of $G$ such that $U_{\alpha}$ and $U_{\alpha^{-1}}$ can be made locally compact (e.g., any expansive automorphism). Then $D_{\alpha} \cap D_{\alpha^{-1}}=\{1\}$.

Proof. Since $D_{\alpha}$ and $D_{\alpha^{-1}}$ are closed and $\alpha$-stable, their intersection $H:=$ $D_{\alpha} \cap D_{\alpha^{-1}}$ is a totally disconnected, locally compact contraction group for both $\left.\alpha\right|_{H}$ and $\left.\alpha^{-1}\right|_{H}$. Hence $H=\{1\}$. Indeed, if $H \neq\{1\}$ then both $\Delta_{H}\left(\left.\alpha\right|_{H}\right)$ and $\Delta_{H}\left(\left.\alpha\right|_{H} ^{-1}\right)=\Delta_{H}\left(\left.\alpha\right|_{H}\right)^{-1}$ would be integers $\geq 2$ (see 1.9(a)), which is impossible.

Remark 5.2 We mention that the nub $U_{0}$ of an expansive automorphism $\alpha: G \rightarrow G$ need not have an open normalizer in $G$. To see this, let $F$ be a finite group which is a semidirect product $F=N \rtimes H$ of a normal subgroup $N$ and a subgroup $H$ which is not normal in $F$ (e.g., $F$ might be the dihedral group $\left.C_{3} \rtimes C_{2}\right)$. Let $G$ be the group of all $\left(n_{k}, h_{k}\right)_{k \in \mathbb{Z}} \in F^{\mathbb{Z}}$ such that $\left(n_{k}\right)_{k \in \mathbb{Z}} \in N^{(-\mathbb{N})} \times N^{\mathbb{N}_{0}}=: M$. Thus $G=M \rtimes H^{\mathbb{Z}}$ as an abstract group. Endow $G$ with the topology making it the direct product topological space of the restricted product $M$ and the compact group $H^{\mathbb{Z}}$. Then $G$ is a topological group, being the ascending union of the open subgroups

$$
H^{\{k \in \mathbb{Z}: k<-m\}} \times F^{\{k \in \mathbb{Z}: k \geq-m\}}
$$


for $m \in \mathbb{N}$, which are topological groups. The right-shift $\alpha$ is an automorphism of $G$. We have $U_{\alpha}=M \rtimes\left(H^{(-\mathbb{N})} \times H^{\mathbb{N}_{0}}\right)$ and $U_{\alpha^{-1}}=H^{-\mathbb{N}} \times H^{\left(\mathbb{N}_{0}\right)}$. Thus $\overline{U_{\alpha}}=G, \overline{U_{\alpha^{-1}}}=H^{\mathbb{Z}}$ and $U_{0}=\overline{U_{\alpha}} \cap \overline{U_{\alpha^{-1}}}=H^{\mathbb{Z}}$ (using $1.2(\mathrm{~b})$ ). Since $H$ is not normal in $F$, we see that $U_{0}=H^{\mathbb{Z}}$ is not normal in $G$. If the normalizer $N_{G}\left(U_{0}\right)$ was open in $G$, then (being $\alpha$-stable), it would contain the dense subgroup $U_{\alpha}$ of $G$ and hence coincide with $G$ (a contradiction). Thus $N_{G}\left(U_{0}\right)$ is not open.

\section{Abelian expansion groups}

We show that, after passing to a refinement if necessary, only abelian, nondiscrete groups of a special form will occur in Theorem B.

Remark 6.1 In the situation of Theorem B, let $I$ be the set of all indices $j \in\{1, \ldots, n\}$ such that $G_{j-1} / G_{j}$ is abelian and non-discrete. Let $\alpha_{j}$ be the automorphism of $G_{j-1} / G_{j}$ induced by $\alpha$ and $q_{j}: G_{j-1} \rightarrow G_{j-1} / G_{j}$ be the quotient homomorphism, for $j \in I$. Then $U_{\alpha_{j}} U_{\alpha_{j}^{-1}}$ is an open $\alpha_{j}$-stable subgroup of $G_{j-1} / G_{j}$ and hence $H_{j}:=q_{j}^{-1}\left(U_{\alpha_{j}} U_{\alpha_{j}^{-1}}\right)$ is an $\alpha$-stable open normal subgroup of $G_{j-1}$. Then $G_{j-1} / H_{j}$ is discrete and all stable, closed, proper subgroups of $H_{j} / G_{j}$ are discrete. After inserting the $H_{j}$ into the series for all $j \in I$, we may thus assume without loss of generality that all abelian, non-discrete subfactors $G_{j-1} / G_{j}$ have the property that all of their $\alpha_{j}$-stable, closed, proper subgroups are discrete, and that $G_{j-1} / G_{j}=U_{\alpha_{j}} U_{\alpha_{j}^{-1}}$.

Let $G_{j}$ be a topological group and $\alpha_{j} \in \operatorname{Aut}\left(G_{j}\right)$ for $j \in\{1,2\}$. We say that $\left(G_{1}, \alpha_{1}\right)$ and $\left(G_{2}, \alpha_{2}\right)$ are isomorphic if there exists an isomorphism $\phi: G_{1} \rightarrow G_{2}$ of topological groups such that $\alpha_{2} \circ \phi=\phi \circ \alpha_{1}$.

Proposition 6.2 Let $A \neq\{1\}$ be an abelian, totally disconnected, locally compact group and $\alpha: A \rightarrow A$ be an expansive automorphism. Assume that $A=U_{\alpha} U_{\alpha^{-1}}$ and assume that every $\alpha$-stable proper closed subgroup of $A$ is discrete. Then there exists a prime number $p$ such that $(A, \alpha)$ isomorphic to one of the following:

(a) $\mathbb{Q}_{p}^{n}$ for some $n \in \mathbb{N}$, together with a contractive linear automorphism $\beta: \mathbb{Q}_{p}^{n} \rightarrow \mathbb{Q}_{p}^{n}$ not admitting non-trivial proper $\beta$-stable vector subspaces;

(b) $\mathbb{Q}_{p}^{n}$ for some $n \in \mathbb{N}$, together with $\beta^{-1}$ for a contractive linear automorphism $\beta: \mathbb{Q}_{p}^{n} \rightarrow \mathbb{Q}_{p}^{n}$ not admitting non-trivial proper $\beta$-stable vector subspaces; 
(c) $C_{p}^{(-\mathbb{N})} \times C_{p}^{\mathbb{N}_{0}}$ with the right-shift;

(d) $C_{p}^{(-\mathbb{N})} \times C_{p}^{\mathbb{N}_{0}}$ with the left-shift;

(e) $C_{p}^{\mathbb{Z}}$ with the right-shift.

Proof. Let $D_{\alpha}$ be the divisible part and $T_{\alpha}$ be the torsion part of $U_{\alpha}$, and define $D_{\alpha^{-1}}$ and $T_{\alpha^{-1}}$ analogously. If $D_{\alpha} \neq\{1\}$, then $D_{\alpha}=D_{\alpha}^{*}$ is an $\alpha$-stable closed subgroup (see Theorem C) which is non-discrete (see 1.8(a)) and thus $A=D_{\alpha}$. By 1.8 (a) and the hypotheses, $D_{\alpha}$ is a divisible simple contraction group and hence of the form described in (a) (see [10, Theorem A]). Likewise, $A$ is of the form described in (b) whenever $D_{\alpha^{-1}} \neq\{1\}$.

Throughout the rest of the proof, assume that $D_{\alpha}=D_{\alpha^{-1}}=\{1\}$. Then $A=U_{\alpha} U_{\alpha^{-1}}=T_{\alpha} T_{\alpha^{-1}}$.

Since the nub $U_{0}$ of $\alpha$ is an $\alpha$-stable closed subgroup of $A$, it either is all of $A$ or discrete. Being also compact, it is finite in the latter case, and thus $\{1\}$ is an open $\alpha$-stable (normal) subgroup of $U_{0}$. Now [31, Corollary 4.4] (proper such do not exist) shows that $U_{0}=\{1\}$.

Case $U_{0}=\{1\}$ : Then $T_{\alpha}=U_{\alpha}=U_{\alpha} U_{0}$ and $T_{\alpha^{-1}}=U_{\alpha^{-1}}=U_{\alpha^{-1}} U_{0}$ are closed $\alpha$-stable subgroups of $A$ (using [1.2(a)). If $T_{\alpha} \neq\{1\}$, then $T_{\alpha}$ is nondiscrete. Hence $T_{\alpha}=A$ by the hypotheses, and this is a simple contraction group which is a torsion group and hence of the form described in (c) (see [10. Theorem A]). Likewise, $A$ is of the form described in (d) if $T_{\alpha^{-1}} \neq\{1\}$.

Case $A=U_{0}$ : Then $A$ is compact and is irreducible in the sense of [31, Definition 6.1] as all its proper $\alpha$-stable closed (normal) subgroups are finite, and moreover $A$ is infinite (as $U_{\alpha}$ or $U_{\alpha^{-1}}$ is non-trivial and hence nondiscrete, being a contraction group). Hence, by [31, Proposition 6.3], $(A, \alpha)$ is isomorphic to the right-shift of $F^{\mathbb{Z}}$ for a finite simple group $F$. Since $A$ is abelian, $F \cong C_{p}$ for some $p$ and thus $A$ is of the form described in (e).

Remark 6.3 Let $G$ be a totally disconnected, locally compact group and $\alpha: G \rightarrow G$ be an expansive automorphism. If $G$ is abelian, then the map

$$
\pi: U_{\alpha}^{*} \times U_{\alpha^{-1}}^{*} \rightarrow G, \quad(x, y) \mapsto x y
$$

is a continuous, open homomorphism with discrete kernel. For non-abelian $G$, the map still has open image (see Lemma 1.1(d)), is a local homeomorphism, and equivariant with respect to the natural left and right actions of $U_{\alpha}$ and 
$U_{\alpha^{-1}}$, respectively.

[To see this, let $V \subseteq G$ be a compact open subgroup such that $V_{+} \cap V_{-}=\{1\}$ and $V=V_{+} V_{-}$(see Lemma 1.1(c)). Then $V_{-}$and $V_{+}$are open subgroups of $U_{\alpha}^{*}$ and $U_{\alpha^{-1}}^{*}$, respectively (see proof of Lemma 2.3). Then $\pi\left(V_{+} \times V_{-}\right)=V$ is open in $G$ and $\left.\pi\right|_{V_{+} \times V_{-}}$is injective, as $v w=v^{\prime} w^{\prime}$ for $v, v^{\prime} \in V_{+}, w, w^{\prime} \in V_{-}$ implies $v^{-1} v^{\prime}=w\left(w^{\prime}\right)^{-1} \in V_{+} \cap V_{-}=\{1\}$ and thus $v=v^{\prime}$ and $w=w^{\prime}$. Since $V_{+} \times V_{-}$is compact, $\pi$ restricts to a homeomorphism $V_{+} \times V_{-} \rightarrow V$. Since $\pi(g v, w h)=g \pi(v, w) h$ for $g \in U_{\alpha}, h \in U_{\alpha^{-1}}$ and $(v, w) \in V_{+} \times V_{-}$, also $\left.\pi\right|_{g V_{+} \times V_{-} h}$ is a homeomorphism onto an open set.]

Remark 6.4 It can happen that $U_{\alpha}$ is closed for an expansive automorphism $\alpha$ of a totally disconnected, locally compact group $G$, but $U_{\bar{\alpha}}$ is not closed for the induced automorphism $\bar{\alpha}$ on $G / N$ for some $\alpha$-stable closed normal subgroup $N \subseteq G$. The following example also illustrates Remark 6.3.

Given a non-trivial finite abelian group $(F,+)$, consider the restricted products $H_{1}:=F^{(-\mathbb{N})} \times F^{\mathbb{N}_{0}}$ and $H_{2}:=F^{-\mathbb{N}} \times F^{\left(\mathbb{N}_{0}\right)}$, with $V_{1}:=F^{\mathbb{N}_{0}}$ and $V_{2}:=F^{-\mathbb{N}}$, respectively, as compact open subgroups. Let $\alpha$ be the right-shift on $G$ : $=H_{1} \times H_{2}$ (i.e., on both $H_{1}$ and $H_{2}$ ). Then $\alpha$ is an automorphism and it is expansive as $\bigcap_{n \in \mathbb{Z}} \alpha^{n}\left(V_{1} \times V_{2}\right)=\{0\}$. Moreover, $U_{\alpha}=H_{1}$ and $U_{\alpha^{-1}}=H_{2}$ are closed. Also, let $\bar{\alpha}$ be the right-shift on $F^{\mathbb{Z}}$. Then

$$
q: G \rightarrow F^{\mathbb{Z}}, \quad(f, g) \mapsto f+g
$$

is a continuous surjective homomorphism. Restricted to the compact open subgroup $V_{1} \times V_{2}$, the map $q$ is an isomorphism of topological groups. Hence $q$ is open, has discrete kernel, and is a quotient morphism. Finally, $U_{\bar{\alpha}}=$ $F^{(-\mathbb{N})} \times F^{\mathbb{N}_{0}}$ is a dense proper subgroup in $F^{\mathbb{Z}}$. Hence $U_{\bar{\alpha}}$ is not closed in $F^{\mathbb{Z}} \cong G / \operatorname{ker}(q)$.

Another property can be observed.

Proposition 6.5 Let $G$ be a totally disconnected, locally compact group that is abelian, and $\alpha: G \rightarrow G$ be an expansive automorphism. Then the torsion subgroup tor $(G)$ is closed in $G$.

Proof. Since $V:=U_{\alpha} U_{\alpha^{-1}}$ is an open subgroup of $G$, we need only show that $V \cap \operatorname{tor}(G)=\operatorname{tor}(V)$ is closed. After replacing $G$ with its $\alpha$-stable subgroup $V$, we may therefore assume that $G=U_{\alpha} U_{\alpha^{-1}}$. Since $D_{\alpha}$ and 
$D_{\alpha^{-1}}$ are torsion-free (see 1.9(c)) and $D_{\alpha} \cap D_{\alpha^{-1}}=\{1\}$ by Corollary 5.1, we deduce that $D_{\alpha} D_{\alpha^{-1}}$ is isomorphic to $D_{\alpha} \times D_{\alpha^{-1}}$ as an abstract group and hence torsion-free. Hence $D_{\alpha} D_{\alpha^{-1}} \cap T_{\alpha} T_{\alpha^{-1}}=\{1\}$. Combining this with $G=U_{\alpha} U_{\alpha^{-1}}=D_{\alpha} D_{\alpha^{-1}} T_{\alpha} T_{\alpha^{-1}}$, we see that

$$
G=\left(D_{\alpha} D_{\alpha^{-1}}\right) \times\left(T_{\alpha} T_{\alpha^{-1}}\right)=D_{\alpha} \times D_{\alpha^{-1}} \times T_{\alpha} T_{\alpha^{-1}}
$$

internally as an abstract group. By (2), the torsion subgroup of $G$ is $\operatorname{tor}(G)=$ $T_{\alpha} T_{\alpha^{-1}}$. Hence $\operatorname{tor}(G)$ has finite exponent (like $T_{\alpha}$ and $T_{\alpha^{-1}}$ ). Thus also $\overline{\operatorname{tor}(G)}$ is a torsion group (by 1.5) and thus $\operatorname{tor}(G)=\overline{\operatorname{tor}(G)}$.

\section{Example: $p$-adic Lie groups}

Let $\mathbb{K}$ be a local field and |.| be an absolute value on $\mathbb{K}$ defining its topology (see [27]). We pick an algebraic closure $\overline{\mathbb{K}}$ containing $\mathbb{K}$ and use the same symbol, |.|, for the unique extension of the absolute value on $\mathbb{K}$ to an absolute value on $\overline{\mathbb{K}}$ (see [20, Theorem 16.1]). If $E$ is a finite-dimensional $\mathbb{K}$-vector space and $\beta: E \rightarrow E$ a $\mathbb{K}$-linear automorphism, we write $R(\beta)$ for the set of all absolute values $|\lambda|$ of zeros $\lambda$ of the characteristic polynomial of $\beta$ in $\overline{\mathbb{K}}$. We let $\widetilde{E}_{\lambda} \subseteq E \otimes_{\mathbb{K}} \overline{\mathbb{K}}$ be the generalized eigenspace of $\beta \otimes_{\mathbb{K}}$ id $\overline{\mathbb{K}}_{\overline{\mathbb{K}}}$ for the eigenvalue $\lambda$. For $\rho \in R(\beta)$, we let

$$
E_{\rho}:=\left(\bigoplus_{|\lambda|=\rho} \widetilde{E}_{\lambda}\right) \cap E .
$$

Then $E=\bigoplus_{\rho \in R(\beta)} E_{\rho}$ (see [17, Chapter II, §1]) and we recall that

$$
E=U_{\beta} \oplus M_{\beta} \oplus U_{\beta^{-1}}
$$

with

$$
M_{\beta}=E_{1}, \quad U_{\beta}=\bigoplus_{\rho<1} E_{\rho} \quad \text { and } \quad U_{\beta^{-1}}=\bigoplus_{\rho>1} E_{\rho}
$$

(cf. [8, Lemma 2.5]).

If $G$ is a Lie group over $\mathbb{K}$, then its tangent space $L(G):=T_{1}(G)$ at the identity element carries a natural Lie algebra structure, and $L(\alpha): L(G) \rightarrow$ $L(H)$ is a Lie algebra homomorphism for each $\mathbb{K}$-analytic homomorphism $\alpha: G \rightarrow H$ between $\mathbb{K}$-analytic Lie groups. We abbreviate $\operatorname{Ad}(g):=L\left(I_{g}\right)$, where $I_{g}: G \rightarrow G, x \mapsto g x g^{-1}$ for $g \in G$ (cf. [23] for further information). 
Proposition 7.1 Let $\alpha$ be an analytic automorphism of a Lie group $G$ over a local field. Then the following conditions are equivalent:

(a) $\alpha$ is expansive;

(b) $\beta:=L(\alpha): L(G) \rightarrow L(G)$ is expansive;

(c) $1 \notin R(\beta)$.

If $\alpha$ is expansive, then $L(G)$ is a nilpotent Lie algebra.

Proof. $(\mathrm{a}) \Rightarrow(\mathrm{b})$ : By contraposition. If (b) is false, then $\beta$ is not expansive. To deduce that $\alpha$ is not expansive, let $V \subseteq G$ be an identity neighbourhood. Since $U_{\beta}$ is a vector subspace of $L(G)$ by (3) and hence closed, using Proposition 1.3 (b) we deduce that $M_{\beta}$ is not discrete and hence a non-trivial vector subspace (in view of (3) ). But then $G$ contains a so-called centre manifold $W$ around the fixed point 1 of $\alpha$, which can be chosen as a submanifold of $G$ contained in $V$ that is stable under $\alpha$ and satisfies $T_{1}(W)=M_{\alpha}$ (whence $W \neq\{1\}$ ); see Proposition 6.3 (a) and part (b) of the Local Invariant Manifold Theorem in [8]; cf. also [7]. Then $\{1\} \neq W \subseteq \bigcap_{n \in \mathbb{Z}} \alpha^{n}(V)$, and thus $\alpha$ is not expansive.

(b) $\Leftrightarrow(\mathrm{c})$ : Since $U_{\beta}$ is closed, $\beta$ is expansive if and only if $M_{\beta}=L(G)_{1}$ is discrete. Since $L(G)_{1}$ is a vector space, the latter holds if and only if $L(G)_{1}=\{0\}$, i.e., $1 \notin R(\beta)$.

$(\mathrm{c}) \Rightarrow(\mathrm{a}): \quad U_{\alpha}$ and $U_{\alpha^{-1}}$ are immersed Lie subgroups of $G$ with Lie algebras $U_{\beta}$ and $U_{\beta^{-1}}$, respectively (see [8, Theorem D]). We write $U_{\alpha}^{*}$ for $U_{\alpha}$ as a Lie group; because the underlying topology is locally compact and $\alpha$ restricts to a contractive Lie group automorphism, this is consistent with the definition of $U_{\alpha}^{*}$ in Section 2. Likewise, we consider $U_{\alpha^{-1}}^{*}$ as a Lie group. If $1 \notin R(\beta)$, then $L(G)=U_{\beta} \oplus U_{\beta^{-1}}$, entailing that the product map $\pi: U_{\alpha}^{*} \times U_{\alpha^{-1}}^{*} \rightarrow G,(x, y) \mapsto x y$ has invertible differential at $(1,1)$ (the addition map $\left.U_{\beta} \times U_{\beta^{-1}} \rightarrow L(G)=U_{\beta} \oplus U_{\beta^{-1}}\right)$. Thus, by the inverse function theorem [23], there exist open identity neighbourhoods $V \subseteq U_{\alpha}^{*}$ and $W \subseteq U_{\alpha^{-1}}^{*}$ such that $V W$ is open in $G$ and the restriction

$$
\left.\pi\right|_{V \times W}: V \times W \rightarrow V W
$$

is an analytic diffeomorphism. Using [24, Lemma $3.2(\mathrm{i})$ ], we find compact open subgroups $P \subseteq V$ of $U_{\alpha}^{*}$ and $Q \subseteq W$ of $U_{\alpha^{-1}}^{*}$ such that $\alpha(P) \subseteq P$, 
$\alpha^{-1}(P) \subseteq V, \alpha^{-1}(Q) \subseteq Q$ and $\alpha(Q) \subseteq W$. Then $P Q$ is an open identity neighbourhood in $G$ and we now show that $\bigcap_{n \in \mathbb{Z}} \alpha^{n}(P Q)=\{1\}$. To this end, let $x \in P$ and $y \in Q$. If $x \neq 1$, then $\alpha^{-n}(x) \notin P$ for some $n \in \mathbb{N}$, which we choose minimal. Thus $\alpha^{-n+1}(x) \in P$ and hence $\alpha^{-n}(x) \in V$. Since $\alpha^{-n}(x) \in V \backslash P$ by the preceding and $\alpha^{-n}(y) \in Q$, we see that $\alpha^{-n}(x y)=$ $\alpha^{-n}(x) \alpha^{-n}(y) \in(V \backslash P) Q$. As the map (44) is a bijection, we deduce that $\alpha^{-n}(x y) \notin P Q$. Likewise, $\alpha^{m}(x y) \notin P Q$ for some $m \in \mathbb{N}$ if $y \neq 1$. Thus $\bigcap_{n \in \mathbb{Z}} \alpha^{n}(P Q)=\{1\}$ indeed and thus $\alpha$ is expansive.

Final assertion. If (c) holds, then $\beta$ is a Lie algebra automorphism of $L(G)$ and $|\lambda| \neq 1$ for all eigenvalues $\lambda$ of $\beta \otimes_{\mathbb{K}} \operatorname{id}_{\overline{\mathbb{K}}}$ in $\overline{\mathbb{K}}$, entailing that none of the $\lambda$ is a root of unity. Hence $L(G)$ is nilpotent (see Exercise 21 (b) among the exercises for Part I of [3], $\S 4$, or [12, Theorem 2]).

7.2 For each continuous homomorphism $\theta: \mathbb{Q}_{p} \rightarrow \mathrm{GL}_{n}\left(\mathbb{Q}_{p}\right)$, there exists a nilpotent $n \times n$-matrix $x \in \mathbb{Q}_{p}^{n \times n}$ such that $\theta(t)=\exp (t x)$ for all $t \in \mathbb{Q}_{p}$, using the matrix exponential function [19, Theorem 1.1]. Thus $\theta^{\prime}(0)=x$ uniquely determines $\theta$, and so does $\left.\theta\right|_{W}$ for any 0-neighbourhood $W \subseteq \mathbb{Q}_{p}$.

Lemma 7.3 Let $\alpha$ be a contractive automorphism of a p-adic Lie group $G$. Then the following holds:

(a) For each $g \in G$, there is a unique continuous homomorphism $\theta_{g}$ : $\mathbb{Q}_{p} \rightarrow$ $G$ such that $\theta_{g}(1)=g$. Moreover, $\left\{\theta_{g}^{\prime}(0): g \in G\right\}=L(G)$.

(b) If $\mathfrak{h} \subseteq L(G)$ is an $L(\alpha)$-stable Lie subalgebra, then there exists an $\alpha$ stable Lie subgroup $H$ of $G$ with $L(H)=\mathfrak{h}$.

Proof. (a) Let $*: L(G) \times L(G) \rightarrow L(G)$ be the Campbell-Hausdorff multiplication on the nilpotent Lie algebra $L(G)$. Because $(G, \alpha)$ and $((L(G), *), L(\alpha))$ are locally isomorphic contraction groups, they are isomorphic (see [26, Proposition 2.2]). The nilpotent group $(L(G), *)$ inherits unique divisibility from $(L(G),+)$, since $n g$ (in the vector space $L(G)$ ) coincides with $g^{n}$ (in $(L(G), *)$ ). It is clear from this that $\theta_{g}(t)=t g$ is the unique continuous homomorphism $\mathbb{Q}_{p} \rightarrow(L(G), *)$ with $\theta_{g}(1)=g$. It satisfies $g=\theta_{g}^{\prime}(0)$.

(b) We may work with the isomorphic group $(L(G), *)$ instead of $G$. Now $H:=\mathfrak{h}$ is an $L(\alpha)$-stable Lie subgroup of $(L(G), *)$ with Lie algebra $\mathfrak{h}$. 
Lemma 7.4 Let $G$ be a linear p-adic Lie group. Assume that $G$ is generated by $\bigcup_{\theta \in \Theta} \theta\left(\mathbb{Q}_{p}\right)$ for a set $\Theta$ of continuous homomorphisms $\theta: \mathbb{Q}_{p} \rightarrow G$, and $L(G)$ is generated by $\left\{\theta^{\prime}(0): \theta \in \Theta\right\}$ as a Lie algebra. Then the centre of $G$ coincides with the kernel of $\operatorname{Ad}: G \rightarrow \operatorname{Aut}(L(G))$.

Proof. Let $g \in G$. For each $\theta \in \Theta$, the map $I_{g} \circ \theta: \mathbb{Q}_{p} \rightarrow G, t \mapsto g \theta(t) g^{-1}$ is a continuous homomorphism such that $\left(I_{g} \circ \theta\right)^{\prime}(0)=\operatorname{Ad}(g) \theta^{\prime}(0)$. Thus, by 7.2. $I_{g} \circ \theta=\theta$ if and only if $\operatorname{Ad}(g) \theta^{\prime}(0)=\theta^{\prime}(0)$. Since $\bigcup_{\theta \in \Theta} \theta\left(\mathbb{Q}_{p}\right)$ generates $G$, we see that $g \in Z(G)$ if and only if $\operatorname{Ad}(g) \theta^{\prime}(0)=\theta^{\prime}(0)$ for all $\theta \in \Theta$. The latter is equivalent to $\operatorname{Ad}(g)(x)=x$ for all $x \in L(G)$, because $\{x \in L(G): \operatorname{Ad}(g)(x)=x\}$ is a Lie subalgebra of $L(G)$ and $L(G)$ is generated by $\theta^{\prime}(0)$ for $\theta \in \Theta$ by hypothesis.

Proof of Theorem D. After replacing $G$ with an open subgroup, we may assume that $G$ is generated by $U_{\alpha} \cup U_{\alpha^{-1}}$ (see Lemma 1.1(d)). We prove that $G$ is nilpotent in this case, by induction on the $\operatorname{dimension} \operatorname{dim}(G)$ of $G$ as a $p$-adic manifold. If $\operatorname{dim}(G)=0$, then $G$ is discrete, whence $U_{\alpha}=U_{\alpha^{-1}}=$ $\{1\}$ and $G=\left\langle U_{\alpha} \cup U_{\alpha^{-1}}\right\rangle=\{1\}$ is nilpotent.

Now assume that $\operatorname{dim}(G)>0$. After replacing $G$ with an isomorphic group, we may assume that $G$ is a subgroup of $\mathrm{GL}_{n}\left(\mathbb{Q}_{p}\right)$ for some $n \in \mathbb{N}$, and that the inclusion map $G \rightarrow \mathrm{GL}_{n}\left(\mathbb{Q}_{p}\right)$ is continuous (but not necessarily a homeomorphism onto its image). Then $L(G)$ is a non-zero nilpotent Lie algebra (see $(\mathrm{a}) \Rightarrow(\mathrm{d})$ in Proposition 7.1) and so it has centre $Z(L(G)) \neq\{0\}$. The centre is $L(\alpha)$-stable, and the restriction $\beta$ of $L(\alpha)$ to the centre is expansive (like $L(\alpha))$. Hence $Z(L(G))=U_{\beta} \oplus U_{\beta^{-1}}$. After replacing $\alpha$ with $\alpha^{-1}$ if necessary, we may assume that $U_{\beta^{-1}} \neq\{0\}$. According to Lemma [7.3(b), there is an $\alpha$-stable Lie subgroup $H \subseteq U_{\alpha^{-1}}$ with $L(H)=U_{\beta}$. We claim that $H$ is in the centre $Z(G)$ of $G$. If this is true, then $Z(G)$ has positive dimension. Thus $G / Z(G)$ is a Lie group of $\operatorname{dimension} \operatorname{dim}(G / Z(G))<\operatorname{dim}(G)$, and it is a linear Lie group as it injects into $\operatorname{Aut}(L(G))$, by Lemma 7.4$]^{3}$ By induction, $G / Z(G)$ is nilpotent and hence so is $G$.

To prove the claim, let $h \in H$ and $\theta: \mathbb{Q}_{p} \rightarrow H \subseteq U_{\alpha^{-1}}$ be a continuous homomorphism with $\theta(1)=h$ (see Lemma 7.3(a)). Then $x:=\theta^{\prime}(0) \in$

\footnotetext{
${ }^{3}$ Let $\Theta$ be the set of continuous homomorphisms from $\mathbb{Q}_{p}$ to $U_{\alpha}$ or $U_{\alpha^{-1}}$. By $G=$ $\left\langle U_{\alpha} \cup U_{\alpha^{-1}}\right\rangle$ and Lemma 7.3(a), the first hypothesis of Lemma 7.4 is satisfied. Since $L(G)=L\left(U_{\alpha}\right)+L\left(U_{\alpha^{-1}}\right)$ and $L\left(U_{\alpha}\right) \cup L\left(U_{\alpha^{-1}}\right)=\left\{\theta^{\prime}(0): \theta \in \Theta\right\}$ by Lemma 7.3. (a) and "(a) $\Rightarrow(\mathrm{c})$ " in Lemma 7.1, also the second hypothesis of Lemma 7.4 is satisfied.
} 
$L(H) \subseteq Z(L(G))$, entailing that $\operatorname{ad}(x):=[x, \bullet]=0$. Now $\theta(t)=\exp (t x)$ for all $t \in \mathbb{Q}_{p}$, by 7.2 . For $|t|$ small, $\operatorname{Ad}(\theta(t))=\operatorname{Ad}(\exp (t x))=e^{t \operatorname{ad}(x)}=\operatorname{id}_{L(G)}$, using Corollary 3 in [3. Chapter III, §4, no.4]). Thus $\operatorname{Ad} \circ \theta=\operatorname{id}_{L(G)}$, by the uniqueness assertion of 7.2 , applied to $\operatorname{Ad} \circ \theta: \mathbb{Q}_{p} \rightarrow \operatorname{Aut}(L(G))$. In particular, $\operatorname{Ad}(h)=\operatorname{Ad}(\theta(1))=\operatorname{id}_{L(G)}$ and thus $h \in Z(G)$, by Lemma 7.4. $\square$

7.5 If $G$ is a totally disconnected, locally compact group which is a nilpotent group, let $\{1\}=Z_{0} \triangleleft Z_{1} \triangleleft \cdots \triangleleft Z_{n}=G$ be its ascending central series defined recursively via $Z_{k}:=q_{k}^{-1}\left(Z\left(G / Z_{k-1}\right)\right)$, where $q_{k}: G \rightarrow G / Z_{k-1}$ is the canonical quotient morphism. Let $\alpha$ be an expansive automorphism of $G$ and $\alpha_{k}$ the induced automorphism of $G_{k} / G_{k-1}$.

Proposition 7.6 If $Z_{k} / Z_{k-1}=U_{\alpha_{k}} U_{\alpha_{k}^{-1}}$ for all $k \in\{1, \ldots, n\}$ in the situation of [7.5, then $G=U_{\alpha} U_{\alpha^{-1}}$. In particular, $U_{\alpha} U_{\alpha^{-1}}$ is a subgroup of $G$.

Proof. If $n=0$, then $G=\{1\}=U_{\alpha} U_{\alpha^{-1}}$. If $n \geq 1$, let $\beta$ be the expansive automorphism of $G / Z(G)$ induced by $\alpha$, and $q: G \rightarrow G / Z(G)$ be the canonical quotient morphism. Then $Z_{1}=Z(G)=\left(U_{\alpha} \cap Z(G)\right)\left(U_{\alpha^{-1}} \cap Z(G)\right)$ by the hypotheses and $G / Z(G)=U_{\beta} U_{\beta^{-1}}$ by induction. Since $q\left(U_{\alpha} U_{\alpha^{-1}}\right)=U_{\beta} U_{\beta^{-1}}=$ $G / Z(G)$, we have $G=U_{\alpha} U_{\alpha^{-1}} Z(G)=U_{\alpha} U_{\alpha^{-1}}\left(U_{\alpha} \cap Z(G)\right)\left(U_{\alpha^{-1}} \cap Z(G)\right)=$ $U_{\alpha}\left(U_{\alpha} \cap Z(G)\right) U_{\alpha^{-1}}\left(U_{\alpha^{-1}} \cap Z(G)\right)=U_{\alpha} U_{\alpha^{-1}}$.

Note that we can easily achieve that $G / Z_{n-1}=U_{\alpha_{n}} U_{\alpha_{n}^{-1}}$ after replacing $G$ with its open subgroup generated by $U_{\alpha} \cup U_{\alpha^{-1}}$. However, the hypotheses on $Z_{k} / Z_{k-1}$ for $k<n$ cannot always be achieved by passing to an open subgroup (as the following example illustrates).

Remark 7.7 The following example shows that even for nilpotent $p$-adic Lie groups with an expansive automorphism $\alpha$, the set $U_{\alpha} U_{\alpha^{-1}}$ may fail to be a subgroup. The example also provides a $p$-adic Lie group that admits expansive automorphisms but does not admit any contractive automorphism. In fact, the group has a closed discrete commutator group which is characteristic and hence would inherit a contractive automorphism (contradicting the fact that non-trivial contraction groups are non-discrete).

Let $H=\mathbb{Q}_{p}^{3}$ be the 3 -dimensional $p$-adic Heisenberg group whose binary operation is given by

$$
\left(x_{1}, y_{1}, z_{1}\right)\left(x_{2}, y_{2}, z_{2}\right)=\left(x_{1}+x_{2}, y_{1}+y_{2}, z_{1}+z_{2}+x_{1} y_{2}\right)
$$


for all $\left(x_{1}, y_{1}, z_{1}\right),\left(x_{2}, y_{2}, z_{2}\right) \in H$. Let $N=\{(0,0, z) \in H:|z| \leq 1\}$. Then $N$ is a compact central subgroup of $H$. Identify $G=H / N$ with $\mathbb{Q}_{p} \times \mathbb{Q}_{p} \times$ $\left(\mathbb{Q}_{p} / \mathbb{Z}_{p}\right)$ as a set. Define $\alpha: G \rightarrow G$ by

$$
\alpha\left(x, y, z+\mathbb{Z}_{p}\right)=\left(p x, p^{-1} y, z+\mathbb{Z}_{p}\right)
$$

for all $\left(x, y, z+\mathbb{Z}_{p}\right) \in G$. Then $\alpha$ is a continuous automorphism of the $p$-adic Lie group $G$ with $M_{\alpha}=\left\{\left(0,0, z+\mathbb{Z}_{p}\right): z \in \mathbb{Q}_{p}\right\}, U_{\alpha}=\left\{(x, 0,0): x \in \mathbb{Q}_{p}\right\}$ and $U_{\alpha^{-1}}=\left\{(0, y, 0): y \in \mathbb{Q}_{p}\right\}$. Since $M_{\alpha}$ is discrete, $\alpha$ is an expansive automorphism. As $\left[U_{\alpha}, U_{\alpha^{-1}}\right]=\left\{\left(0,0, z+\mathbb{Z}_{p}\right): z \in \mathbb{Q}_{p}\right\}$ and $U_{\alpha} U_{\alpha^{-1}}=$ $\left\{\left(x, y, x y+\mathbb{Z}_{p}\right): x, y \in \mathbb{Q}_{p}\right\}$, we get that $U_{\alpha} U_{\alpha^{-1}}$ is a not a subgroup.

Proposition 7.8 Let $G$ be a closed subgroup of $\mathrm{GL}_{n}\left(\mathbb{Q}_{p}\right)$ and $\alpha$ be an expansive automorphism of $G$. Then $U_{\alpha} U_{\alpha^{-1}}$ is an open (unipotent algebraic) subgroup of $G$.

Proof. Replacing $G$ by the group generated by $U_{\alpha}$ and $U_{\alpha^{-1}}$, we may assume by Theorem $\mathrm{E}$ that $G$ is a closed nilpotent subgroup of $\mathrm{GL}_{n}\left(\mathbb{Q}_{p}\right)$. Let $\mathbb{G}$ be the Zariski closure of $G$. Then $\mathbb{G}$ is defined over $\mathbb{Q}_{p}$ and $\mathbb{G}$ is nilpotent (cf. Proposition 1.3 (b) and Corollary 1 in 2.4 of [2]). Since $U_{\alpha}$ and $U_{\alpha^{-1}}$ consists of one-parameter (unipotent) subgroups, $\mathbb{G}$ is Zariski-connected. This implies that the set of unipotent elements form a subgroup $\mathbb{G}_{u}$, known as the unipotent radical (cf. Theorem 10.6 of [2]). Since $U_{\alpha}$ and $U_{\alpha^{-1}}$ consists of one-parameter (unipotent) subgroups, $U_{\alpha}, U_{\alpha^{-1}} \subseteq \mathbb{G}_{u}$. This implies that $\mathbb{G}=\mathbb{G}_{u}$, that is $\mathbb{G}$ is an unipotent algebraic group, hence $\mathbb{G}$ is defined over $\mathbb{Q}_{p}$ (cf. 4.5 of [2] and the fact that $\mathbb{Q}_{p}$-closed and defined over $\mathbb{Q}_{p}$ are same as characteristic of $\mathbb{Q}_{p}$ is zero) and $G \subseteq \mathbb{G}\left(\mathbb{Q}_{p}\right)$.

For $i \geq 1$, let $D_{i}=\left[\mathbb{G}\left(\mathbb{Q}_{p}\right), D_{i-1}\right]$ with $D_{0}=\mathbb{G}\left(\mathbb{Q}_{p}\right)$ and $G_{i}=\overline{\left[G, G_{i-1}\right]}$ with $G_{0}=G$. Then $D_{k+1}$ is trivial for some $k \geq 1$ as $\mathbb{G}$ is unipotent and $G_{i} \subset D_{i}$. Thus, $G_{k}$ is a closed $\alpha$-stable subgroup of $D_{k}$ which is a vector space. Let $V$ be the maximal vector subspace of $G_{k}$. Then $V$ is a closed $\alpha$-stable central subgroup of $G$. The automorphism $\beta: G_{k} / V \rightarrow G_{k} / V$ defined by $\beta(x+V)=\alpha(x)+V$ for $x \in G_{k}$ is expansive. Since $V$ is the maximal vector subspace of $G_{k}$ and $G_{k}$ is a closed subgroup of the p-adic vector space $D_{k}$, we get that $G_{k} / V$ is a compact subgroup of the $p$-adic vector space $D_{k} / V$. Since the automorphism group of a compact $p$-adic analytic group is compact, compact $p$-adic analytic groups do not admit expansive automorphisms unless finite, hence $V=G_{k}$. This implies that $G_{k}=D_{k}$ and 
$G_{k}=V=\left(U_{\alpha} \cap V\right)\left(U_{\alpha^{-1}} \cap V\right)$. Since $G / G_{k}$ is a closed subgroup of $\mathbb{G}\left(\mathbb{Q}_{p}\right) / D_{k}$ which is a linear ( $p$-adic algebraic) group, the result follows by induction.

Remark 7.9 In the case of linear $p$-adic Lie groups, even if $U_{\alpha} U_{\alpha^{-1}}$ is an open subgroup for an expansive automorphism, the following example shows that it is not possible to have either of $U_{\alpha}$ or $U_{\alpha^{-1}}$ to normalize the other.

Let $H$ be the 3 -dimensional $p$-adic Heisenberg group defined as in Remark 7.7 . For $i=1,2$, define $\alpha_{i}: H \rightarrow H$ by

$$
\alpha_{1}(x, y, z)=\left(p x, p^{-2} y, p^{-1} z\right), \quad \alpha_{2}(x, y, z)=\left(p^{2} x, p^{-1} y, p z\right)
$$

for $(x, y, z) \in H$. Let $G=H \times H$ and $\alpha=\alpha_{1} \times \alpha_{2}$. Then

$$
U_{\alpha}=\left\{(x, 0,0): x \in \mathbb{Q}_{p}\right\} \times\left\{(a, 0, c): a, c \in \mathbb{Q}_{p}\right\}
$$

and

$$
U_{\alpha^{-1}}=\left\{(0, y, z): y, z \in \mathbb{Q}_{p}\right\} \times\left\{(0, b, 0) \mid b \in \mathbb{Q}_{p}\right\} .
$$

Thus $U_{\alpha} U_{\alpha^{-1}}=G$. Since $\left\{(x, 0,0): x \in \mathbb{Q}_{p}\right\}$ and $\left\{(0, y, 0): y \in \mathbb{Q}_{p}\right\}$ are not normal subgroups of $H$, neither $U_{\alpha}$ or $U_{\alpha^{-1}}$ normalize the other.

\section{Example: Baumslag-Solitar groups}

Throughout this section, we fix primes $p \neq q$. We let

$$
\operatorname{BS}(p, q):=\left\langle a, t \mid t a^{p} t^{-1}=a^{q}\right\rangle
$$

be the Baumslag-Solitar group. Then $\langle a\rangle \cap g\langle a\rangle g^{-1}$ has finite index in $\langle a\rangle$ for each $g \in \operatorname{BS}(p, q)$, and $\bigcap_{g} g\langle a\rangle g^{-1}=\{1\}$, hence the Schlichting completion $G_{p, q}$ of $\operatorname{BS}(p, q)$ can be formed, which is a certain totally disconnected, locally compact group in which $\mathrm{BS}(p, q)$ is dense, and in which $K:=\overline{\langle a\rangle}$ is a compact open subgroup (see [4], cf. [21] and [9]). We are interested in the inner automorphism

$$
\alpha: G_{p, q} \rightarrow G_{p, q}, \quad x \mapsto t x t^{-1} .
$$

Proof of Theorem E. By [4, Proposition 8.1], $K$ contains an open subgroup $V \cong \mathbb{Z}_{p} \times \mathbb{Z}_{q}$ and $K / V$ is a cyclic group of order diving $\operatorname{gcd}(p, q)=1$. Thus $K=V \cong \mathbb{Z}_{p} \times \mathbb{Z}_{q}$. After multiplication with a unit, We may assume that the isomorphism takes $a$ to $(1,1)$. 
Let $G=\mathbb{Z} \ltimes\left(\mathbb{Q}_{p} \times \mathbb{Q}_{q}\right)$ be the semidirect product of $\mathbb{Z}$ and $\mathbb{Q}_{p} \times \mathbb{Q}_{q}$ given by $(n, u, v)\left(m, u^{\prime}, v^{\prime}\right)=\left(n+m, u+(q / p)^{n} u^{\prime}, v+(q / p)^{n} v^{\prime}\right)$ for all $n, m \in \mathbb{Z}, u, u^{\prime} \in$ $\mathbb{Q}_{p}$ and $v, v^{\prime} \in \mathbb{Q}_{q}$. The isomorphism $K \cong \mathbb{Z}_{p} \times \mathbb{Z}_{q}$ gives a homomorphism from $\langle a\rangle$ to $G$. Since $(1,0,0)(0, p, p)(-1,0,0)=(0, q, q)$, sending $t \mapsto(1,0,0)$ yields a group homomorphism $\phi: \operatorname{BS}(p, q) \rightarrow G$. Since $\left.\phi\right|_{\langle a\rangle}$ is a continuous homomorphism, $\phi$ extends to a continuous homomorphism of $G_{p, q}$ into $G$ which would also be denoted by $\phi$. Since $\left.\phi\right|_{K}$ is an isomorphism, $\operatorname{ker}(\phi)$ is discrete. Moreover, as $\phi\left(G_{p, q}\right)$ contains both $(1,0,0)$ and $\mathbb{Z}_{p} \times \mathbb{Z}_{q}, \phi$ is surjective.

Let $\beta$ be the inner automorphism of $G$ given by $(1,0,0)$. Then $\phi \circ \alpha=\beta \circ \phi$ and $\beta$ is expansive. Since the kernel of $\phi$ is discrete, expansiveness of $\alpha$ follows from Theorem A. As the open subgroup $K \cong \mathbb{Z}_{p} \times \mathbb{Z}_{q}$ satisfies an ascending chain condition on closed subgroups (see, e.g., [6, Proposition 3.2]), $U_{\alpha}$ is closed by [26, Lemma 3.2].

In case $U_{\alpha} U_{\alpha^{-1}}$ is a group, we will now show that $\phi$ is an isomorphism which would lead to a contradiction as $G_{p, q}$ is not solvable but $G$ is solvable 4 Suppose $N:=U_{\alpha} U_{\alpha^{-1}}$ is a group. Then $\left.\phi\right|_{N}$ is an isomorphism of $N$ with $\mathbb{Q}_{p} \times \mathbb{Q}_{q}$ (using that $U_{\alpha} \cong \mathbb{Q}_{q}$ and $\left.U_{\alpha^{-1}} \cong \mathbb{Q}_{p}\right)$.5 Now the group generated by $t$ and $N$ is an open subgroup of $G$ containing both $t$ and $a$, hence $G_{p, q}=$ $\langle t, N\rangle=\langle t\rangle N$ (as $t$ normalizes $N$ ). This implies that $\phi$ is an isomorphism.

\section{References}

[1] Baumgartner, U. and G.A. Willis, Contraction groups and scales of automorphisms of totally disconnected locally compact groups, Israel J. Math. 142 (2004), 221-248.

[2] Borel, A., "Linear algebraic groups". Second edition. Graduate Texts in Mathematics, 126. Springer-Verlag, New York, 1991.

[3] Bourbaki, N., "Lie Groups and Lie Algebras, Chapters 1-3," Springer-Verlag, Berlin, 1989.

[4] Elder, M. and G. A. Willis, Totally disconnected groups from Baumslag-Solitar groups, preprint, arXiv:1301.4775.

\footnotetext{
${ }^{4}$ Alternatively, $\operatorname{BS}(p, q)$ would be a finitely generated linear group then and hence residually finite by [16]. But $\operatorname{BS}(p, q)$ is not residually finite for primes $p \neq q$, see [18.

${ }^{5}$ In fact, $V=K$ is tidy for $\alpha$ with $V_{-} \cong \mathbb{Z}_{q}, V_{+} \cong \mathbb{Z}_{p}$ and $V_{0}=\{1\}$ (see [4]), whence $U_{\alpha}=V_{--} \cong \mathbb{Q}_{q}$ and $U_{\alpha^{-1}}=V_{++} \cong \mathbb{Q}_{p}$ (cf. Lemma 1.1(b).
} 
[5] Glöckner, H., Contraction groups for tidy automorphisms of totally disconnected groups, Glasg. Math. J. 47 (2005), 329-333.

[6] Glöckner, H., Locally compact groups built up from p-adic Lie groups, for $p$ in a given set of primes, J. Group Theory 9 (2006), 427-454.

[7] Glöckner, H., Invariant manifolds for analytic dynamical systems over ultrametric fields, Expo. Math. 31 (2013), 116-150.

[8] Glöckner, H., Invariant manifolds for finite-dimensional non-archimedean dynamical systems, in: H. Glöckner, A. Escassut and K. Shamseddine (eds.), "Advances in Non-Archimedean Analysis," to appear in Contemp. Math.

[9] Glöckner, H. and G. A. Willis, Topologization of Hecke pairs and Hecke $C^{*}$ algebras, Topol. Proc. 26 (2001-2002), 565-591.

[10] Glöckner, H. and G. A. Willis, Classification of the simple factors appearing in composition series of totally disconnected contraction groups, J. Reine Angew. Math. 643 (2010), 141-169.

[11] Hewitt, E. and K. A. Ross, "Abstract Harmonic Analysis," Vol. I, Springer, 2nd Edition, New York, 1979.

[12] Jacobson, N., A note on automorphisms and derivations of Lie algebras, Proc. Amer. Math. Soc. 6 (1955), 281-283.

[13] Jaworski, W., On contraction groups of automorphisms of totally disconnected locally compact groups, Israel J. Math. 172 (2009), 1-8.

[14] Kitchens, B.P., Expansive dynamics on zero-dimensional groups, Ergodic Theory Dynam. Systems 7 (1987), 249-261.

[15] Lam, P., On expansive transformation groups, Trans. Amer. Math. Soc. 150 (1970), 131-138.

[16] Mal'cev, A.I., On the faithful representation of infinite groups by matrices. Transl. Amer. Math. Soc. (2), 45 (1965), 1-18.

[17] Margulis, G. A., "Discrete Subgroups of Semisimple Lie Groups," Springer, Berlin, 1991.

[18] Meskin, S., Nonresidually finite one-relator groups, Trans. Amer. Math. Soc. 164 (1972), 105-114.

[19] Ratner, M., Raghunathan's conjectures for Cartesian products of real and $p$ adic Lie groups, Duke Math. J. 77 (1995), 275-382.

[20] Schikhof, W.H., "Ultrametric Calculus,"Cambridge University Press, 1984. 
[21] Schlichting, G., On the periodicity of group operations, pp. 507-517 in: Cheng, Kai Nah and Leong, Yu Kiang (eds.), "Group Theory" (Singapore 1987), de Gruyter, Berlin, 1989.

[22] Schmidt, K., "Dynamical systems of algebraic origin," Progress in Mathematics, 128. Birkhäuser Verlag, Basel, 1995.

[23] Serre, J.-P., "Lie Algebras and Lie Groups," Springer, Berlin, 1992.

[24] Siebert, E., Contractive automorphisms on locally compact groups, Math. Z. 191 (1986), 73-90.

[25] Siebert, E., Semistable convolution semigroups and the topology of contraction groups, pp.325-343 in: "Probability Measures on Groups, IX" (Oberwolfach, 1988), Lecture Notes in Math. 1379, Springer, Berlin, 1989.

[26] Wang, J.S.P., The Mautner phenomenon for p-adic Lie groups, Math. Z. 185 (1984), 403-412.

[27] Weil, A., "Basic Number Theory," Springer, Berlin, 2nd ed., 1973.

[28] Wesolek, P., Elementary totally disconnected locally compact groups, Proc. Lond. Math. Soc. (3) 110 (2015), 1387-1434.

[29] Willis, G.A., The structure of totally disconnected, locally compact groups, Math. Ann. 300 (1994), 341-363.

[30] Willis, G. A., Further properties of the scale function on a totally disconnected group, J. Algebra 237 (2001), 142-164.

[31] Willis, G. A., The nub of an automorphism of a totally disconnected, locally compact group, Ergodic Theory Dyn. Syst. 34 (2014), 1365-1394.

Helge Glöckner, Universität Paderborn, Institut für Mathematik, Warburger Str. 100, 33098 Paderborn, Germany; e-mail: glockner@math.upb.de

C. R. E. Raja, Stat-Math Unit, Indian Statistical Institute (ISI), 8th Mile Mysore Road, Bangalore 560 059, India; e-mail: creraja@isibang. ac.in 